\title{
MATHEMATICAL DETERMINATION OF THE FRÉCHET DERIVATIVE WITH RESPECT TO THE DOMAIN FOR A FLUID-STRUCTURE SCATTERING PROBLEM. CASE OF POLYGONAL-SHAPED DOMAINS.
}

\author{
HÉLÈNE BARUCQ*, RABIA DJELLOULI ${ }^{\dagger}$, ELODIE ESTECAHANDY ${ }^{\ddagger}$, AND MOHAND \\ MOUSSAOUI§
}

\begin{abstract}
The characterization of the Fréchet derivative of the elasto-acoustic scattered field with respect to Lipschitz continuous polygonal domains is established. The considered class of domains is of practical interest since two-dimensional scatterers are always transformed into polygonalshaped domains when employing finite element methods for solving direct and inverse scattering problems. The obtained result indicates that the Fréchet derivative with respect to the scatterer of the scattered field is the solution of the same elasto-acoustic scattering problem but with additional right-hand side terms in the transmission conditions across the fluid-structure interface. This characterization has the potential to advance the state-of-the-art of the solution of inverse obstacle problems.
\end{abstract}

Key words. Scattering, Elasto-acoustic, Helmholtz equation, Navier's equation, Shape derivative, Fréchet derivative, Inverse problem

AMS subject classifications. 35B65, 35J05, 35Q60, 35R30, 65N21, 74J20, 78A45

1. Introduction. One of the basic inverse scattering problems in scattering theory is the determination of the shape of the scatterer using some measured scattered far-field patterns [8]. This model problem, called inverse obstacle problem (IOP), is relevant to numerous real-world applications including radar and sonar detection, geophysical exploration, structural design, medical imaging, and atmospheric studies. In spite of their apparent simple formulations, IOPs are very challenging problems from both mathematical and computational viewpoints. The difficulties in studying and/or solving IOPs are mainly due to their nonlinear and severely ill-posed nature [8]. Nevertheless, given the applied nature of these problems and their prevalence in sciences and engineering, IOPs have been subject to extensive studies leading to a tremendous growth within the last three decades with an emphasis on the development of computational methods (see, for example, [11, 14], and the references therein).

A large class of the proposed solution methodologies for IOPs belongs to the category of regularized Newton-type algorithms [21, 17]. These methods appear to be naturally well-suited for solving IOPs for two reasons. First, the Newton component of these algorithms is the primary candidate for addressing the nonlinear aspect of IOPs. Second, the regularization procedure is incorporated to restore the stability to these problems. Employing this class of regularized iterative strategies requires

*INRIA Bordeaux Sud-Ouest Research Center, Project-Team Magique-3D, LMA - UMR CNRS 5142, Université de Pau et des Pays de l'Adour, Avenue de l'Université, BP 1155, 64013 PAU Cedex, FRANCE (helene.barucq@inria.fr).

$\dagger$ Interdisciplinary Research Institute for the Sciences (IRIS) \& INRIA Associate Team Magic, Department of Mathematics, California State University Northridge, 18111 Nordhoff Street, CA 91330 Northridge, USA (rabia.djellouli@csun.edu).

${ }^{\ddagger}$ INRIA Bordeaux Sud-Ouest Research Center, Project-Team Magique-3D, LMA - UMR CNRS 5142, Université de Pau et des Pays de l'Adour, Avenue de l'Université, BP 1155, 64013 PAU Cedex, FRANCE (elodie.estecahandy@alumni.univ-pau.fr).

$\S$ Ecole Normale Supérieure, Dept of Mathematics, B.P.92, 16308 Vieux-Kouba, Alger, ALGERIA (mmohand47@gmail.com.). 
however the computation, at each Newton-type iteration, of the Fréchet derivatives of the scattered field with respect to the shape parameters at each given observation point. Furthermore, it is well-known that the accuracy, fast convergence, and computational efficiency of these iterative algorithms strongly depend on the accuracy and effectiveness levels in the calculation of the Jacobians that occur at each iteration. Hence, characterizing these Fréchet derivatives is a necessary condition for solving efficiently and accurately IOPs with regularized iterative algorithms. It is therefore not surprising to note that a great deal of attention, documented by numerous publications in the literature, was given to address this issue. Indeed, depending on the regularity assumptions on the shape of the considered scatterer, various approaches were suggested to establish and characterize the Fréchet differentiability of the scattered field with respect to the shape of the scatterer. Studies and results pertaining to the case of acoustic scattering problems by rigid (not penetrable) scatterers can be found, for example, in $[29,24,35,37,25,12]$. The case of electromagnetic scattering problems has been also studied by several authors and results can be found in [36, 21, 9, 10, 30], among other references. The case of elasto-acoustic problems was recently partially addressed in [7]. It was proved that the elasto-acoustic scattered field and its corresponding far-field pattern are continuously Fréchet differentiable with respect to the elastic domain. This important preliminary result was established assuming the boundary of the considered elastic scatterer and its perturbations to be Lipschitz continuous only. The proof of this result is a two-step approach based on the ideas developed in [33, 40]. In the first step, the direct elasto-acoustic problem is reformulated in carefully constructed Banach spaces that are invariant with respect the the shape perturbations. The second step is devoted to proving that the conditions for applying the Implicit Function Theorem are satisfied. It is worth mentioning that [7] does not indicate how this derivative can be computed/characterized. Note that this new result extends the one established in [13] to elastic object with rough boundaries that are usually encountered in practical problems.

Characterizing the Fréchet derivative of the elasto-acoustic field with respect to the domain is a delicate task that requires a particular attention. More specifically, for elastic scatterers that are regular enough (of class $\mathcal{C}^{k} ; k \geq 2$ ), it is relatively simple to prove that the derivative is the solution of the same boundary value problem but with different right-hand sides on the transmission conditions across the fluid-structure interface (see Theorem 3.2 in Section 5.1). The proof of this result is straightforward and relies on the chain rule along with the classical trace theorems [34, 31, 19]. The case of Lipschitz continuous elastic domains, that are of practical interest, is more complicated due to the presence of second-order derivatives of the elasto-acoustic field in the transmission conditions (see Theorem 3.2). The presence of these higher-order derivatives is problematic because it requires to define in an intrinsic way the Sobolev space $H^{3 / 2}(\Gamma)$ and its corresponding dual space. In the case of a Lipschitz continuous boundary, it is well-known that in general it is not possible to define $H^{s}(\Gamma)$ and its dual except for $s \in[-1,1]$. The underlying difficulty is that the multiplication by an $L^{\infty}(\Gamma)$-function is not a continuous mapping from $H^{1 / 2}(\Gamma)$ into itself, which constitutes an irremediable obstruction. It is worth mentioning that Geymonat and Krasucki provided in reference [16] a characterization of the space of the traces for functions in $H^{2}(\Omega)$ as a subspace of $H^{1}(\Gamma)$. Unfortunately, the approach employed to establish this result cannot be used for clearly characterizing the dual space. We must point out that we previously announced in an extended abstract that appeared in the special issue [5] that the main result of this paper is valid in the case of "pure" 
Lipschitz domains. Neither the derivation of this characterization nor the proof were included in the paper due to space limitations. It turns out that the unpublished proof was not correct. Indeed, one of the main intermediate trace results was not valid for "pure" Lipschitz domains. We unfortunately are no longer convinced that it would be possible to characterize the derivative of the scattered field as the solution of an elasto-acoustic-type problem when the boundary $\Gamma$ is assumed to be Lipschitz continuous only. Note that this property is true in the case of non penetrable obstacles, i.e. acoustic scattering problems [12]. For this reason, we limit our study to the class of Lipschitz curvilinear polygonal-shaped domains. This assumption encompasses a large class of domains since, at the computational level, all scatterer's shapes are transformed, after applying finite-element triangulations, into polygonalshaped domains. The main advantage in considering this class of Lipschitz continuous domains is that the unit normal vector to the domain's boundary $\Gamma$ is well-defined and remains -at least- Lipschitzian everywhere except possibly at the vertices of the considered polygon. This allows to adopt a "divide \& conquer" type strategy to prove Theorem 3.2. More specifically, we adopt a two-step approach to establish this characterization. In the first step, we perform a local analysis, i.e. at the edges level of $\Gamma$. The goal in the second step of the proposed proof is to extend the local results to the entire boundary $\Gamma$. This is accomplished through the derivation of two new key trace results (see Proposition 4.9 and Proposition 4.10) that also appear to be of independent interest. The computational implication of this theoretical characterization is that, at each Newton iteration, one need to solve a single linear system of equations with $N_{p}$ right-hand-sides; $N_{p}$ being the number of parameters representing the surface of the considered scatterer. This result has the potential to advance the state-of-the-art of the solution of inverse elasto-acoustic scattering problems. Indeed, the methodology adopted for characterizing the Fréchet derivatives with respect to the shape of an elastic scatterer can also be applied to analyze the Fréchet differentiability with respect to its material properties. This is relevant to many inverse problems where not only the shape of an obstacle is of interest but also, and equally important, its material properties. To the best of our knowledge, this class of inverse elasto-acoustic problems has not been solved yet. Last, we must point out that employing adjoint-based formulations could be an attractive alternative for solving inverse obstacle elasto-acoustic problems. One of the main advantages of this class of methods is that the derivatives are expressed for all perturbations which makes this class of methods independent of the number of the shape parameters. In addition, these methods require solving one forward problem and its corresponding adjoint problem. However, since the resulting linear system corresponding to the direct problem is symmetric but not hermitian, its factorization cannot be reusable to solve the adjoint problem. Hence, the computational cost associated with the assembly and the factorization of two linear systems could be superior to the one required for the solution of one linear system with multiple right-hand sides.

The remainder of the paper is organized as follows. In Section 2, we specify the nomenclature and assumptions adopted, and formulate the considered elasto-acoustic scattering problem [28]. In Section 3, we specify the considered class of Lipschitz continuous domains and state the main result of this paper: Theorem 3.2. This result shows that the Fréchet derivative with respect to the domain of the elasto-acoustic scattered field is a solution of the same elasto-acoustic scattering problem but with different right-hand sides in the transmission conditions across the wet interface $\Gamma$. Section 4 is devoted to the derivation of preliminary trace results that form the math- 
ematical framework of the proposed study. Some of these results do not appear to be standard and they may be of independent interest. The proof of Theorem 3.2 is given in Section 5. We first prove this theorem in the case where the boundary $\Gamma$ is regular enough (Section 5.1). Then, we extend the proof to the case of Lipschitz continuous polygonal-shaped domains (Section 5.2). Concluding remarks are presented in Section 6.

\section{Problem Statement.}

2.1. Nomenclature and Assumptions. Throughout this paper, we adopt the following notations and assumptions:

- $\Omega^{s}$ is a polygonal-shaped domain of $\mathbb{R}^{2}$ whose boundary $\Gamma$ is assumed to be Lipschitz continuous.

- $\Omega^{f}=\mathbb{R}^{2} \backslash \bar{\Omega}^{s}$ is the homogeneous inviscid (fluid) medium surrounding the elastic domain.

- $|\cdot|$ is the Euclidean norm in $\mathbb{R}^{2}$.

- $x$ is a point of $\mathbb{R}^{2}$, understood as a column vector and $r=|x|$ is the distance from an origin point to $x$.

- $d$ is a unit vector representing the propagation direction of the incident plane wave.

- $\nu$ is the outward normal to $\Gamma$ and $\frac{\partial}{\partial \nu}$ is the normal derivative operator.

- $S^{1}=\left\{x \in \mathbb{R}^{2}|| x \mid=1\right\}$ is the unit sphere in $\mathbb{R}^{2}$.

- $\nabla$ is the gradient operator in $\mathbb{R}^{2}$. The gradient of a scalar function is a column vector field. We adopt the notations from Simon-Murat [33] for the Jacobian matrix. The gradient of a column vector field is a matrix, where the partial derivatives are listed in rows. For a row vector field, we also define the gradient as a matrix, where the partial derivatives are the columns entries.

- $\nabla \cdot$ is the divergence operator in $\mathbb{R}^{2}$.

- $\Delta$ is the Laplace operator in $\mathbb{R}^{2}$.

- $\mathcal{D}(E)$ is the space of infinitely differentiable functions with compact support in $E$.

- $\mathcal{D}^{\prime}(E)$ is the standard space of distributions [1].

- $\mathcal{C}^{0,1}(E)$ is the set of all Lipschitz continuous functions on $E \subset \mathbb{R}^{2}$, equipped with the norm $\|\phi\|_{\mathcal{C}^{0,1}(E)}=\|\phi\|_{\mathcal{C}^{0}(E)}+|\phi|_{L i p(E)}$, where $|\phi|_{L i p}=\sup _{x_{1} \neq x_{2}} \mid \phi\left(x_{1}\right)-$ $\phi\left(x_{2}\right)|/| x_{1}-x_{2} \mid$.

- $\mathcal{C}^{1}(E)$ is the space of functions with continuous derivatives on $E \subset \mathbb{R}^{2}$.

- $\mathcal{C}^{m}(E)$ is the space of functions with continuous derivatives up to order $m$ on $E \subset \mathbb{R}^{2}$, with the maximum norm of all derivatives.

- $L^{2}(E)$ is the standard Lebesgue space [1].

- For a bounded domain $E \subset \mathbb{R}^{2}$ and for $m \in \mathbb{N}$, the standard Sobolev spaces are $H^{m}(E)$ [1]. 
- $L_{l o c}^{2}(E)$ and $H_{l o c}^{m}(E)$ are the spaces of functions that are respectively in $L^{2}(D)$ and $H^{m}(D)$ for any open bounded set $D$ in $E$.

- $H_{0}^{1}(E)$ denotes the space of functions of $H^{1}(E)$ that vanish on the boundary $\partial E$, that is,

$$
H_{0}^{1}(E)=\left\{w \in H^{1}(E), w_{\mid \partial E}=0\right\} .
$$

- $H^{s}(\Gamma)$ are the trace Sobolev spaces [1].

- $H^{-s}(\Gamma)$ are the dual spaces of the trace Sobolev spaces in the regular case equipped with the dual product $\langle\cdot, \cdot\rangle_{-s, s, \Gamma}[1]$.

- $I: \mathbb{R}^{2} \rightarrow \mathbb{R}^{2}$ is the identity mapping or the unit matrix.

- $\theta: \mathbb{R}^{2} \rightarrow \mathbb{R}^{2}$ denotes an admissible perturbation in $\left(\mathcal{C}^{1}\left(\mathbb{R}^{2}\right)\right)^{2}$. It is assumed to have a compact support and to be small enough so that $I+\theta$ is bijective in $\mathbb{R}^{2}$, for example $\|\theta\|_{\left(\mathcal{C}^{1}\left(\mathbb{R}^{2}\right)\right)^{2}}<\frac{1}{2}$.

- $\Omega_{\theta}^{\bullet}=(I+\theta) \Omega^{\bullet}$ is an admissible perturbed configuration of the reference domain $\Omega^{\bullet} ; \bullet=s, f$. Note that $\Omega_{0}^{\bullet}=\Omega^{\bullet}$.

- $\Gamma_{\theta}$ is the boundary of $\Omega_{\theta}^{s}$ and $\nu_{\theta}$ is the outward normal to $\Gamma_{\theta}$. Note that $\Gamma_{0}=\Gamma$.

- $\left[\theta^{\prime}\right]$ is the Jacobian matrix of $\theta$. Hence $\left[\theta^{\prime}\right]=\left(\frac{\partial \theta_{l}}{\partial x_{j}}\right)_{1 \leq l, j \leq 2}$.

- $\left[\theta^{\prime}\right]^{t}$ is the transpose Jacobian matrix of $\theta$.

- $\operatorname{Tr}_{\Gamma}$ is the trace operator on $\Gamma$.

- $D_{1}$, and $D_{2}$ are two bounded domains with smooth boundaries, such that

$$
\bar{D}_{1} \subset \Omega^{s} \subset \bar{\Omega}^{s} \subset D_{2} .
$$

- The symbol $\hookrightarrow$ denotes continuous injection between two normed spaces.

- We say that $\theta \mapsto u_{\theta}$ is locally differentiable if for every open set $D$ strictly included in $\Omega^{s}$ and strictly included in $\Omega_{\theta}^{s}$ the restriction of $u_{\theta}$ to $D$ is differentiable [33, 40]. Similarly, in the unbounded domain, we say that $\theta \mapsto p_{\theta}$ is locally differentiable if for every open set $D$ strictly included in $\Omega^{f}$ and strictly included in $\Omega_{\theta}^{f}$ the restriction of $p_{\theta}$ to $D$ is differentiable. We denote by $\left(u^{\prime}, p^{\prime}\right)=\left(\left(\partial u_{\theta} / \partial \theta\right)(0) h,\left(\partial p_{\theta} / \partial \theta\right)(0) h\right)$ the local derivative of $\left(u_{\theta}, p_{\theta}\right)$ at $\theta=0$ in the direction $h$, where $h$ is a vector field satisfying $h \in\left(\mathcal{C}^{2}\left(\mathbb{R}^{2}\right)\right)^{2}$.

2.2. Mathematical Formulation of the Problem. The scattering of a timeharmonic acoustic wave by an elastic obstacle $\Omega_{\theta}^{s}$ embedded in a homogeneous medium $\Omega_{\theta}^{f}$, depicted in Figure 2.1, can be formulated as the following boundary value problem [28]:

$$
\operatorname{BVP}(1)\left\{\begin{array}{rll}
\nabla \cdot \sigma\left(u_{\theta}\right)+\omega^{2} \rho_{s} u_{\theta}=0 & \text { in } \Omega_{\theta}^{s} & (a) \\
\Delta p_{\theta}+k^{2} p_{\theta}=0 & \text { in } \Omega_{\theta}^{f} & (b) \\
\tau\left(u_{\theta}\right)=-p_{\theta} \nu_{\theta}-g \nu_{\theta} & \text { on } \Gamma_{\theta} & (c) \\
\omega^{2} \rho_{f} u_{\theta} \cdot \nu_{\theta}=\frac{\partial p_{\theta}}{\partial \nu_{\theta}}+\frac{\partial g}{\partial \nu_{\theta}} & \text { on } \Gamma_{\theta} & (d) \\
\lim _{r \rightarrow+\infty} \sqrt{r}\left(\frac{\partial p_{\theta}}{\partial r}-\mathrm{i} k p_{\theta}\right)=0 . &
\end{array}\right.
$$




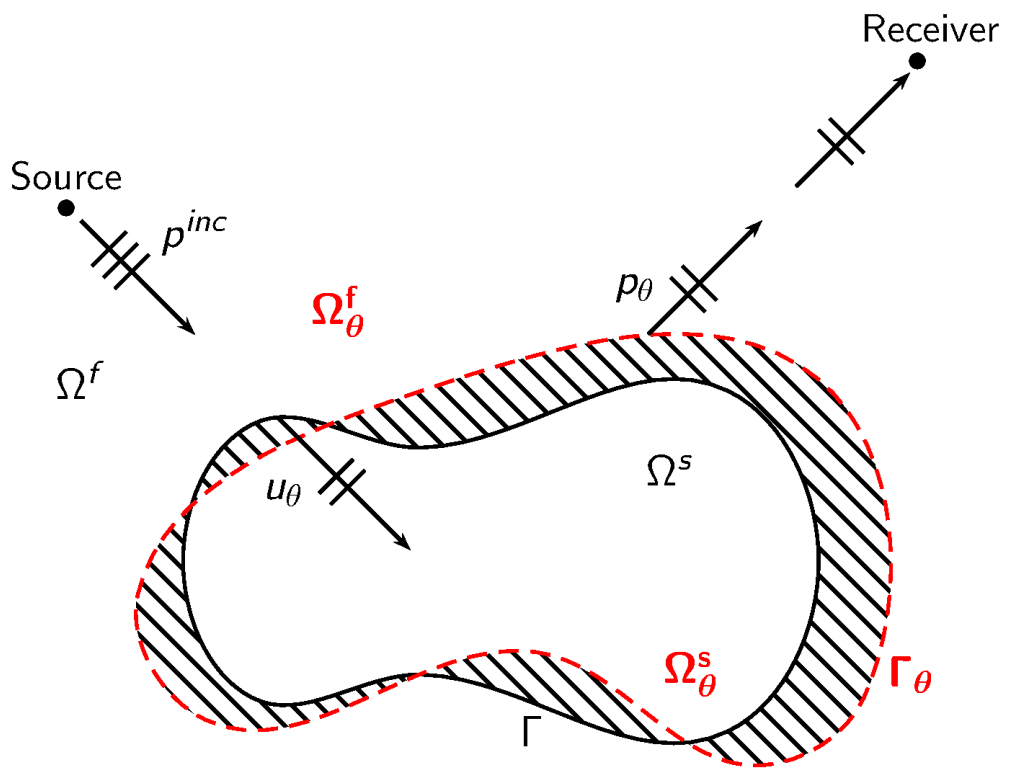

FIG. 2.1. An admissible perturbed configuration $\Omega_{\theta}^{\bullet}$ of the reference domain $\Omega^{\bullet}$, where $\bullet$ is either $s$ or $f$.

where the pair $\left(u_{\theta}, p_{\theta}\right)$ represents the elasto-acoustic scattered field vector. $p_{\theta}$ is the scalar-valued fluid pressure in $\Omega_{\theta}^{f}$, whereas $u_{\theta}$ is the vector-valued displacement field in $\Omega_{\theta}^{s} . g=p^{i n c}=\mathrm{e}^{\mathrm{i} \frac{\omega}{c_{f}} x \cdot d}$ corresponds to the given incident plane wave. $\omega$ is the angular frequency and $c_{f}$ is the speed of sound in the fluid $\Omega_{\theta}^{f} . \omega$ and $c_{f}$ are associated with the wavenumber $k$ by $k=\frac{\omega}{c_{f}}$. $\rho_{f}$ (resp. $\rho_{s}$ ) is a positive real number denoting the density of the fluid $\Omega_{\theta}^{f}$ (resp. the scatterer $\Omega_{\theta}^{s}$ ). $\sigma$ is the stress tensor related to the strain tensor $\varepsilon$ by Hooke's law [28]. We consider here the case of an isotropic medium, and assume $\sigma$ to be invariant under rotations and reflections [28]. Consequently, we have:

$$
\sigma\left(u_{\theta}\right)=\lambda \nabla \cdot u_{\theta} I+2 \mu \varepsilon\left(u_{\theta}\right),
$$

where $\varepsilon$ is the strain tensor given by:

$$
\varepsilon\left(u_{\theta}\right)=\frac{1}{2}\left(\nabla u_{\theta}+\left(\nabla u_{\theta}\right)^{t}\right) .
$$

and the Lamé coefficients $\lambda$ and $\mu$ are two positive real numbers. This means that we assume $\Omega_{\theta}^{s}$ to be a homogeneous scatterer.

Last, $\tau$ denotes the traction vector on the surface of the scatterer $\Omega_{\theta}^{s}$, that is,

$$
\tau\left(u_{\theta}\right)=\sigma\left(u_{\theta}\right) \nu_{\theta} .
$$

We recall that the scattering amplitude $p_{\theta, \infty}$ of the acoustic scattered field $p_{\theta}$ that is the solution of BVP (1) is defined on the unit sphere $S^{1}$ and is obtained from the asymptotic behavior of $p_{\theta}$ [8]:

$$
p_{\theta}=\frac{\mathrm{e}^{\mathrm{i} k r}}{\sqrt{r}}\left(p_{\theta, \infty}\left(\frac{x}{r}\right)+O\left(\frac{1}{r}\right)\right) ; \quad r=\|x\|_{2} \rightarrow+\infty .
$$


Recall that the far-field pattern can also be expressed as follows [8]:

$$
p_{\theta, \infty}(\hat{x})=\frac{\mathrm{e}^{\mathrm{i} \pi / 4}}{\sqrt{8 \pi k}} \int_{\Gamma_{\theta}}\left(\mathrm{e}^{\mathrm{i} k \hat{x} \cdot y} \frac{\partial p_{\theta}}{\partial \nu_{\theta}}(y)-\frac{\partial \mathrm{e}^{\mathrm{i} k \hat{x} \cdot y}}{\partial \nu_{\theta}} p_{\theta}(y)\right) d \Gamma_{\theta} .
$$

Observe that the direct problem BVP (1) contains the standard exterior Helmholtz problem given by Eqs. (b) and (e), and the Navier's equation given by Eq. (a) governing the equilibrium of an elastic scatterer. These equations are coupled via the transmission conditions given by Eqs. (c) and (d). The first one is a dynamic interface condition whereas the second one is a kinematic interface condition [28].

This boundary value problem has been investigated mathematically and results pertaining to the existence, uniqueness and regularity can be found in $[28,32,27,22,6]$, among others.

3. Main results. We consider the class of curvilinear polygonal-shaped domains $\Omega^{s}$ whose boundaries $\Gamma$ are globally Lipschitz continuous, as illustrated in Figure 3.2. More specifically, the boundary $\Gamma$ is given by $\Gamma=\bigcup_{j=1}^{M} \Gamma_{j}$, where $\Gamma_{j} ; j=1, \ldots, M$, are the $M$ sides of the polygon $\Omega^{s}$ ordered with $j$ increasing and counterclockwise. The $j^{\text {th }}$ side line $\Gamma_{j}$ is assumed to be of class $\mathcal{C}^{1,1}$. We denote the vertices of the polygon $\Omega^{s}$ by $S_{j} ; j=1, \ldots, M$, and we set $S_{M+1}=S_{1}$, so that $\Gamma_{j}$ is the curve or the straight line joining the vertices $S_{j}$ and $S_{j+1}$, as illustrated in Figure 3.2. Furthermore, we assume that:

$$
\text { (H) }\left\{\begin{array}{l}
\text { at each vertex } S_{j} \text {, the semi-tangents to } \Gamma_{j-1} \text { and } \Gamma_{j} \\
\text { are neither parallel nor forming an angle of } \pi .
\end{array}\right.
$$

Note that under assumption $(H)$, the unit normal vector $\nu$ to $\Gamma$ is well-defined and Lipschitz continuous everywhere except at the vertices $S_{j}$ and $S j+1$. We denote by $\nu_{j}$ the unit normal vector to $\Gamma_{j}$ and we assume the basis $\left\{\tau_{j}, \nu_{j}\right\}$ to have the canonical orientation, where $\tau_{j}$ is the unit tangent vector to $\Gamma_{j}$.

Remark 3.1. The following example illustrates the important role of assumption $(\mathrm{H})$ on the boundary $\Gamma$ for defining the traces on a polygonal-shaped boundary.

Let $g$ be a real-valued function given by:

$$
g(x)=\left\{\begin{array}{lc}
0, & -\frac{1}{2}<x \leq 0 \\
\int_{0}^{x} \frac{d t}{|\ln t|^{1 / 2}}, & 0 \leq x<\frac{1}{2} .
\end{array}\right.
$$

It is easy to verify that $g$ is continuously differentiable on $\left(-\frac{1}{2}, \frac{1}{2}\right)$, and that $g^{\prime}(0)=$ 0 . However, for any positive number $\alpha, g$ cannot be an element of $\mathcal{C}^{1, \alpha}$.

Next, assume that, locally, $g$ represents a curve $\tilde{\Gamma}$ of the boundary $\Gamma$ of $\Omega^{s}$ and consider the function:

$$
u(x, y)=y \quad \text { in } \Omega^{s} .
$$

Observe that $u \in H^{k}$ in the neighborhood of the origin for all $k$. However, the trace of $u$ is not in $H^{3 / 2}(\tilde{\Gamma})$. The latter being defined in the classical and standard intrinsic 
sense. Note that $\tilde{\Gamma}$ is composed of two curves generated by $g$ in $\left(-\frac{1}{2}, 0\right)$ and $\left(0, \frac{1}{2}\right)$ respectively, and both curves are separately of class $\mathcal{C}^{2}$.

Given that, it is important to be very careful when considering the traces on $H^{s}(\Gamma)$ for $s>1$ or $s<-1$ in the case of polygonal-shaped boundaries. Assumption $(\mathrm{H})$ allows to define such traces via a restriction to each side of the boundary and appropriate connections at the end points.

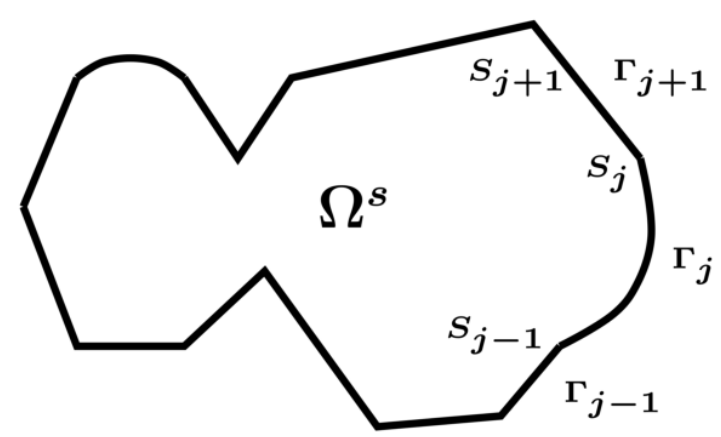

FIG. 3.2. Illustration of a globally Lipschitz continuous polygonal-shaped domain.

The aim of this paper is to establish under assumption $(H)$ the following theorem.

TheOREm 3.2. Let $\left(u^{\prime}, p^{\prime}\right)$ be the local derivative at $\theta=0$ in the direction $h \in$ $\left(\mathcal{C}^{2}\left(\mathbb{R}^{2}\right)\right)^{2}$ of the solution $\left(u_{\theta}, p_{\theta}\right)$ of the problem BVP (1). Then, $\left(u^{\prime}, p^{\prime}\right)$ is the solution of the boundary value problem:

$$
\operatorname{BVP}(2) \begin{cases}\nabla \cdot \sigma\left(u^{\prime}\right)+\omega^{2} \rho_{s} u^{\prime}=0 & \text { in } \Omega^{s} \\ \Delta p^{\prime}+k^{2} p^{\prime}=0 & \text { in } \Omega^{f} \\ \tau\left(u^{\prime}\right)=-p^{\prime} \nu+F(u, p, h) & \text { on } \Gamma \\ \omega^{2} \rho_{f} u^{\prime} \cdot \nu=\frac{\partial p^{\prime}}{\partial \nu}+G(u, p, h) & \text { on } \Gamma \\ \lim _{r \rightarrow+\infty} \sqrt{r}\left(\frac{\partial p^{\prime}}{\partial r}-\mathrm{i} k p^{\prime}\right)=0 & \end{cases}
$$

where the functions $F$ and $G$ are given by:

$$
G(u, p, h)=-\left(\omega^{2} \rho_{f} \nabla u-\nabla\left(\nabla p^{T}\right)\right) h \cdot \nu+\left(\omega^{2} \rho_{f} u-\nabla p^{T}\right) \cdot\left[h^{\prime}\right]^{t} \nu,
$$

and $p^{T}=p+p^{i n c}$. The notation $h^{t} \nabla \sigma(u) \nu$ stands for:

$$
h^{t} \nabla \sigma(u) \nu=\left[h^{t} \nabla \sigma_{l}(u) \nu\right]_{l=1,2},
$$

where $\sigma_{l}$ is the $l^{\text {th }}$ row of the matrix $\sigma(u)$. 
Recall that the mapping $\theta \rightarrow p_{\theta, \infty}$ is continuously Fréchet differentiable, at $\theta=0$ in the direction $h \in\left(\mathcal{C}^{2}\left(\mathbb{R}^{2}\right)\right)^{2}$, from $\left(\mathcal{C}^{1}\left(\mathbb{R}^{2}\right)\right)^{2}$ to $\left(\mathcal{C}^{m}\left(S^{1}\right)\right)^{2}$ (see Corollary 3.2 in [7] or Remark III.3.2.1 in [14]). In addition, the following characterization of the derivative of the far-field pattern $\left(\partial p_{\theta, \infty} / \partial \theta\right)(0) h$ still holds (see [8]).

Corollary 3.3. Let $p_{\infty}^{\prime}$ be the far-field pattern of the solution $p^{\prime}$ of BVP (2), and let $\left(\partial p_{\theta, \infty} / \partial \theta\right)(0) h$ be the derivative at $\theta=0$ in the direction $h \in\left(\mathcal{C}^{2}\left(\mathbb{R}^{2}\right)\right)^{2}$ of the far-field pattern $p_{\theta, \infty}$ of the solution $p_{\theta}$ of $B V P(1)$. We have

$$
\frac{\partial p_{\theta, \infty}}{\partial \theta}(0) h=p_{\infty}^{\prime}
$$

Remark 3.4. Establishing that $p^{\prime}$ (resp. $u^{\prime}$ ) satisfies the Helmholtz (resp. Navier) equation in $\Omega^{f}$ (resp. $\Omega^{s}$ ) does not pose any additional difficulties, as demonstrated in Lemma 5.14. The only part of the proof of Theorem 3.2 that deserves a very careful consideration is the construction of an appropriate mathematical framework in which the transmission conditions in BVP (2) are well-defined because these conditions incur higher-order derivatives of the elasto-acoustic scattered field $(u, p)$ (see Eqs. (3.7)(3.8)). Giving sense to these derivatives in the case where the interface $\Gamma$ is regular enough (e.g. $\left.\mathcal{C}^{2}\right)$ is relatively straightforward, as demonstrated in Section 5.1. This however is no longer the case for polygonal-shaped domains of class $\mathcal{C}^{0,1}$. For the considered class of boundaries, the proof appears to be technical and requires an analysis at a piecewise level, i.e., locally on $\Gamma_{j}$, as shown in Section 5.2. It relies on the characterization of the spaces given by Eqs. (4.10) and (4.16) to give sense to the traces of their functions via the surjectivity of the trace operators in Proposition 4.5 and 4.13 along with the density properties.

4. Auxiliary results. We specify in this section the adopted mathematical framework for the elasto-acoustic scattered field $(u, p)$ and we establish preliminary trace results that are relevant to the proof of Theorem 3.2.

4.1. Mathematical framework for the pressure field and trace properties. Let $D\left(\Delta, \Omega^{f}\right)$ be the maximal domain of the Laplace operator $\Delta$ in the fluid domain $\Omega^{f}$. Hence, $D\left(\Delta, \Omega^{f}\right)$ is given by [19]:

$$
D\left(\Delta, \Omega^{f}\right)=\left\{q \in L_{l o c}^{2}\left(\Omega^{f}\right), \Delta q \in L_{l o c}^{2}\left(\Omega^{f}\right)\right\} .
$$

We also introduce the following Sobolev space:

$$
H^{D}\left(\Omega^{f}\right)=H_{l o c}^{2}\left(\Omega^{f}\right) \cap H_{0}^{1}\left(\Omega^{f}\right) .
$$

Next, for $j=1, \ldots, M$, we consider the following Sobolev space:

$$
H_{j}^{N}\left(\Omega^{f}\right)=\left\{q \in H_{l o c}^{2}\left(\Omega^{f}\right), \frac{\partial q}{\partial \nu}=0 \text { on } \Gamma, q=0 \text { on } \Gamma_{l} \text { for } l \neq j\right\} .
$$

In addition, for $s=1 / 2$ or $3 / 2$, we consider the following trace spaces:

$$
\begin{aligned}
\tilde{H}^{s}\left(\Gamma_{j}\right)=\left\{\varphi \in H^{s}\left(\Gamma_{j}\right) ; \tilde{\varphi} \in H^{s}(\Gamma)\right. & \\
& \text { where } \left.\tilde{\varphi}=\varphi \text { on } \Gamma_{j} \text { and } \tilde{\varphi}=0 \text { on } \Gamma \backslash \Gamma_{j}\right\} .
\end{aligned}
$$

Similarly to the standard notations, the dual space of $\tilde{H}^{s}\left(\Gamma_{j}\right)$ is denoted by $\tilde{H}^{-s}\left(\Gamma_{j}\right)$, and is equipped with the pairing product $\langle\cdot, \cdot\rangle_{\sim,-s, s, \Gamma_{j}}$. 
The following trace results stated as a proposition are immediate consequences of the results established by Grisvard in [19, 20] (see Theorem 1.5.3.4 p.54 and Theorem 1.5.3.6 p.57 in [19]) for polygonal domains of class $\mathcal{C}^{1,1}$. These results have been also extended to Maxwell's system but only for domains with strictly polygonal/polyhedral boundaries in (see $[2,3,4])$.

Proposition 4.5. For $j=1, \ldots, M$, the operators $p \mapsto\left(p_{\mid \Gamma_{j}}, \frac{\partial p}{\partial \nu_{j}} \mid \Gamma_{j}\right)$, that are well-defined on $H_{\text {loc }}^{2}\left(\Omega^{f}\right)$, can be extended to linear and continuous operators from $D\left(\Delta, \Omega^{f}\right)$ into $\tilde{H}^{-1 / 2}\left(\Gamma_{j}\right) \times \tilde{H}^{-3 / 2}\left(\Gamma_{j}\right)$. Furthermore, for any $p \in D\left(\Delta, \Omega^{f}\right)$, we have the following two properties:

$$
\int_{\Omega^{f}} p \Delta \varphi d x-\int_{\Omega^{f}} \Delta p \varphi d x=\sum_{j=1}^{M}\left\langle p, \partial_{\nu_{j}} \varphi\right\rangle_{\sim,-1 / 2,1 / 2, \Gamma_{j}} ; \quad \forall \varphi \in H^{D}\left(\Omega^{f}\right),
$$

$\int_{\Omega^{f}} p \Delta \psi d x-\int_{\Omega^{f}} \Delta p \psi d x=-\left\langle\partial_{\nu_{j}} p, \psi\right\rangle_{\sim,-3 / 2,3 / 2, \Gamma_{j}} ; \quad \forall \psi \in H_{j}^{N}\left(\Omega^{f}\right) ; j=1, \ldots, M$.

Proof. Since $\Gamma$ is a polygonal-shaped boundary satisfying $(\mathrm{H})$, then Eq (4.14) is a direct consequence of Lemmas 3.2 and 3.5 in [18]. To establish Eq (4.15), we first observe that since $\psi \in H_{j}^{N}\left(\Omega^{f}\right)$, then due to assumption $(\mathrm{H})$ and the compatibility conditions of the traces at the vertices, we necessarily have $\psi \in \tilde{H}^{3 / 2}\left(\Gamma_{j}\right)$. Therefore, Eq (4.15) becomes an immediate consequence of the density result given by Theorem 1.1 and Lemma 2.3 in [18].

4.2. Mathematical framework for the displacement field and trace properties. Let $\sigma$ be the stress tensor given by Eq. (2.2) and $D\left(\nabla \cdot \sigma, \Omega^{s}\right)$ be the maximal domain of the operator $\nabla \cdot \sigma$ in the solid domain $\Omega^{s}$. Hence, $D\left(\nabla \cdot \sigma, \Omega^{s}\right)$ is given by [19]:

$$
D\left(\nabla \cdot \sigma, \Omega^{s}\right)=\left\{v \in\left(L^{2}\left(\Omega^{s}\right)\right)^{2}, \nabla \cdot \sigma(v) \in\left(L^{2}\left(\Omega^{s}\right)\right)^{2}\right\} .
$$

The next two lemmas state important properties of the space $D\left(\nabla \cdot \sigma, \Omega^{s}\right)$.

LEMma 4.6. The space $D\left(\nabla \cdot \sigma, \Omega^{s}\right)$ equipped with the scalar product:

$$
(u, v)_{\nabla \cdot \sigma}=\int_{\Omega^{s}} u \cdot v d x+\int_{\Omega^{s}}(\nabla \cdot \sigma(u)) \cdot(\nabla \cdot \sigma(v)) d x
$$

is a Hilbert space.

Proof. Since $D\left(\nabla \cdot \sigma, \Omega^{s}\right)$ is a subspace of the Hilbert space $\left(L^{2}\left(\Omega^{s}\right)\right)^{2}$, it is sufficient to show that $D\left(\nabla \cdot \sigma, \Omega^{s}\right)$ is closed. To this end, let $\left(u_{n}\right)$ be a Cauchy sequence in $D\left(\nabla \cdot \sigma, \Omega^{s}\right)$. Then, $\left(u_{n}\right)$ and $\left(\nabla \cdot \sigma\left(u_{n}\right)\right)$ are also Cauchy sequences in $\left(L^{2}\left(\Omega^{s}\right)\right)^{2}$. Since $\left(L^{2}\left(\Omega^{s}\right)\right)^{2}$ is complete, then there are $u, g \in\left(L^{2}\left(\Omega^{s}\right)\right)^{2}$ such that $u_{n} \underset{n \rightarrow+\infty}{\longrightarrow} u$ and $\nabla \cdot \sigma\left(u_{n}\right) \underset{n \rightarrow+\infty}{\longrightarrow} g$ in $\left(L^{2}\left(\Omega^{s}\right)\right)^{2}$. Consequently, for all $\varphi \in\left(\mathcal{D}\left(\Omega^{s}\right)\right)^{2}$, we have:

$$
\left\langle\nabla \cdot \sigma\left(u_{n}\right), \varphi\right\rangle=\left\langle u_{n}, \nabla \cdot \sigma(\varphi)\right\rangle \underset{n \rightarrow+\infty}{\longrightarrow}\langle u, \nabla \cdot \sigma(\varphi)\rangle=\langle\nabla \cdot \sigma(u), \varphi\rangle=\langle g, \varphi\rangle .
$$

We conclude that $\nabla \cdot \sigma(u)=g \in\left(L^{2}\left(\Omega^{s}\right)\right)^{2}$, and thus $u \in D\left(\nabla \cdot \sigma, \Omega^{s}\right)$. 
Lemma 4.7. $\left(H^{2}\left(\Omega^{s}\right)\right)^{2}$ is dense in $D\left(\nabla \cdot \sigma, \Omega^{s}\right)$ equipped with the graph norm corresponding to the scalar product $(\cdot, \cdot)_{\nabla \cdot \sigma}$ given by Eq. (4.17).

Proof. We prove that $\left(\mathcal{D}\left(\bar{\Omega}^{s}\right)\right)^{2} \subset\left(H^{2}\left(\Omega^{s}\right)\right)^{2}$ is dense in $D\left(\nabla \cdot \sigma, \Omega^{s}\right)$. To this end, we consider a continuous linear form $L$ defined on $D\left(\nabla \cdot \sigma, \Omega^{s}\right)$.

Observe that, for $u \in D\left(\nabla \cdot \sigma, \Omega^{s}\right)$, we have $(u, \nabla \cdot \sigma(u)) \in\left(L^{2}\left(\Omega^{s}\right)\right)^{2} \times\left(L^{2}\left(\Omega^{s}\right)\right)^{2}$. Therefore, $D\left(\nabla \cdot \sigma, \Omega^{s}\right)$ can be identified as a subspace $V$ of $\left(L^{2}\left(\Omega^{s}\right)\right)^{2} \times\left(L^{2}\left(\Omega^{s}\right)\right)^{2}$. Note that $V$ is closed. Thus, using Hahn-Banach theorem (see Chapter IV, Section 5 in [41]), every linear continuous form on $D\left(\nabla \cdot \sigma, \Omega^{s}\right)$ can be associated with a linear form on $V$, that can be extended to a linear continuous form on $\left(L^{2}\left(\Omega^{s}\right)\right)^{2} \times\left(L^{2}\left(\Omega^{s}\right)\right)^{2}$. Consequently, $L$ can be represented as follows:

$$
L(u)=\int_{\Omega^{s}} f \cdot(\nabla \cdot \sigma(u)) d x+\int_{\Omega^{s}} g \cdot u d x
$$

where $f, g \in\left(L^{2}\left(\Omega^{s}\right)\right)^{2}$.

Assume that $L(u)=0$ for all $u \in\left(\mathcal{D}\left(\overline{\Omega^{s}}\right)\right)^{2}$. Then, it follows that:

$$
\langle\nabla \cdot \sigma(f)+g, \varphi\rangle=0 ; \quad \forall \varphi \in\left(\mathcal{D}\left(\overline{\Omega^{s}}\right)\right)^{2} .
$$

Therefore,

$$
\nabla \cdot \sigma(f)+g=0 \quad \text { in }\left(\mathcal{D}^{\prime}\left(\overline{\Omega^{s}}\right)\right)^{2} .
$$

Consequently, all we need to prove is that $L$ vanishes on $D\left(\nabla \cdot \sigma, \Omega^{s}\right)$. To this end, we define $F=\tilde{f}$ and $G=\tilde{g}$ to be the extensions of $f$ and $g$ by zero outside $\Omega^{s}$. Let $U \in\left(\mathcal{D}\left(\mathbb{R}^{2}\right)\right)^{2}$ and $u=U_{\mid \Omega^{s}}$. Then, we have:

$$
L(u)=\int_{\Omega^{s}} g \cdot u d x+\int_{\Omega^{s}} f \cdot \nabla \cdot \sigma(u) d x=\int_{\mathbb{R}^{2}} G \cdot U d x+\int_{\mathbb{R}^{2}} F \cdot(\nabla \cdot \sigma(U)) d x .
$$

Since $L$ vanishes on $\left(\mathcal{D}\left(\overline{\Omega^{s}}\right)\right)^{2}$, we necessarily have:

$$
\int_{\mathbb{R}^{2}} G \cdot P d x+\int_{\mathbb{R}^{2}} F \cdot(\nabla \cdot \sigma(P)) d x=0, \forall P \in\left(\mathcal{D}\left(\mathbb{R}^{2}\right)\right)^{2},
$$

that is,

$$
\nabla \cdot \sigma(F)+G=0 \text { in }\left(\mathcal{D}^{\prime}\left(\mathbb{R}^{2}\right)\right)^{2} .
$$

Hence, we obtain $F \in\left(L^{2}\left(\Omega^{s}\right)\right)^{2}$ and $\nabla \cdot \sigma(F)=-G \in\left(L^{2}\left(\Omega^{s}\right)\right)^{2}$. It follows from the regularity results of elliptic operators [15] that $F \in\left(H^{2}\left(\mathbb{R}^{2}\right)\right)^{2}$.

In addition, it follows from Theorem 4.12 p.90 in [34] that $f \in\left(H_{0}^{2}\left(\Omega^{s}\right)\right)^{2}$. Consequently, we have:

$$
\sigma(f) \nu=0
$$

Using Eq. (4.19), we obtain:

$$
\nabla \cdot \sigma(f)+g=0 \text { in }\left(L^{2}\left(\Omega^{s}\right)\right)^{2} .
$$

Furthermore, there is a sequence $\left(f_{n}\right) \subset\left(\mathcal{D}\left(\Omega^{s}\right)\right)^{2}$ such that (see Theorem 4.12 p.90 in [34]):

$$
f_{n} \underset{n \rightarrow+\infty}{\longrightarrow} f \text { in }\left(H^{2}\left(\Omega^{s}\right)\right)^{2} .
$$


Thus,

$$
\nabla \cdot \sigma\left(f_{n}\right) \underset{n \rightarrow+\infty}{\longrightarrow} \nabla \cdot \sigma(f) \text { in }\left(L^{2}\left(\Omega^{s}\right)\right)^{2} .
$$

Let $u$ be in $D\left(\nabla \cdot \sigma, \Omega^{s}\right)$. We then obtain:

$$
\begin{aligned}
L(u) & =\lim _{n \rightarrow+\infty} \int_{\Omega^{s}}\left(g \cdot u+f_{n} \cdot(\nabla \cdot \sigma(u))\right) d x \\
& =\lim _{n \rightarrow+\infty} \int_{\Omega^{s}}\left(g \cdot u+\left(\nabla \cdot \sigma\left(f_{n}\right)\right) \cdot u\right) d x \\
& =\int_{\Omega^{s}}(g+\nabla \cdot \sigma(f)) \cdot u d x \\
& =0 .
\end{aligned}
$$

This means that every linear continuous form on $D\left(\nabla \cdot \sigma, \Omega^{s}\right)$ that is zero on $\left(\mathcal{D}\left(\overline{\Omega^{s}}\right)\right)^{2}$ must vanish on $D\left(\nabla \cdot \sigma, \Omega^{s}\right)$. This concludes the proof of Lemma 4.7.

Next, for $j=1, \ldots, M$, we set by $\tilde{\mathbf{H}}^{s}\left(\Gamma_{j}\right)=\left(\tilde{H}^{s}\left(\Gamma_{j}\right)\right)^{2}$, where the trace space $\tilde{H}^{s}\left(\Gamma_{j}\right)$ is given by Eq. (4.13). Similarly, we denote by $\tilde{\mathbf{H}}^{-s}\left(\Gamma_{j}\right)$ its corresponding dual space, i.e. $\tilde{\mathbf{H}}^{-s}\left(\Gamma_{j}\right)=\left(\tilde{H}^{-s}\left(\Gamma_{j}\right)\right)^{2}$. Moreover, we define the spaces $\mathbf{H}_{j}^{D}\left(\Omega^{s}\right)$ and $\mathbf{H}_{j}^{N}\left(\Omega^{s}\right)$ as follows:

$$
\begin{aligned}
& \mathbf{H}_{j}^{D}\left(\Omega^{s}\right)=\left\{v \in\left(H^{2}\left(\Omega^{s}\right) \cap H_{0}^{1}\left(\Omega^{s}\right)\right)^{2}, \sigma(v) \nu_{\mid \Gamma_{l}}=0, \forall l \neq j\right\}, \\
& \mathbf{H}_{j}^{N}\left(\Omega^{s}\right)=\left\{v \in\left(H^{2}\left(\Omega^{s}\right)\right)^{2}, \sigma(v) \nu_{\mid \Gamma}=0, v_{\mid \Gamma_{l}}=0, \forall l \neq j\right\} .
\end{aligned}
$$

Next, we state two additional technical results that can also be of independent interest. The first result is a standard result that is formulated as a lemma.

LEMMA 4.8. Consider the open interval $I=(a, b)$ where $a$ and $b$ are two arbitrary real numbers with $a<b$. Let $\varphi$ be a Lipschitz continuous function on $\bar{I}=[a, b]$. Then, the mapping $f \longmapsto \varphi f$ is linear and continuous from $H^{s}(I)$ into $H^{s}(I)$ for $0 \leq s \leq 1$. The second result is a trace result that is formulated as a proposition.

Proposition 4.9. Let $\Omega$ be a polygonal-shaped domain satisfying assumption $(\mathrm{H})$. Then, the operator $T^{1}$ defined by:

$$
\begin{aligned}
T^{1}: H^{2}(\Omega) \cap H_{0}^{1}(\Omega) & \longrightarrow \prod_{j=1}^{M} \tilde{H}^{1 / 2}\left(\Gamma_{j}\right)=\mathbb{P}^{1} \\
u & \longmapsto T^{1} u=\left(\frac{\partial u}{\partial \nu_{1}}\left|\Gamma_{1}, \ldots, \frac{\partial u}{\partial \nu_{M}}\right| \Gamma_{M}\right)^{t}
\end{aligned}
$$

is linear, continuous, and surjective. Moreover, $T^{1}$ admits a linear and continuous operator $R^{1}$ such that:

$$
T^{1} \circ R^{1}=I_{\mathbb{P}^{1}} .
$$

Proof. The result in the case where $\Omega$ is a strictly polygonal-shaped domain is a direct consequence of Theorem 1.1 and Lemma 3.5 in [18].

In the case of curvilinear polygons, we first transform the curvilinear edges into straight lines and then we employ Lemma 4.8 to conclude. 
In the following, we present trace results on the displacement field. These results do not appear to be standard and they may be of independent interest.

Proposition 4.10. For $j=1, \ldots, M$, we have:

(i) The mapping $\gamma_{0, j}: u \mapsto u_{\mid \Gamma_{j}}$ is linear and continuous from $\mathbf{H}_{j}^{N}\left(\Omega^{s}\right)$ into $\tilde{\mathbf{H}}^{3 / 2}\left(\Gamma_{j}\right)$. In addition, $\gamma_{0, j}$ admits a linear and continuous right inverse.

(ii) The mapping $\gamma_{\sigma, j}: u \mapsto \sigma(u) \nu_{\mid \Gamma_{j}}$ is linear and continuous from $\mathbf{H}_{j}^{D}\left(\Omega^{s}\right)$ into $\tilde{\mathbf{H}}^{1 / 2}\left(\Gamma_{j}\right)$. In addition, $\gamma_{\sigma, j}$ admits a linear and continuous right inverse.

Proof. The only property that deserves a special attention is part (ii). Indeed, the proof of part (i) is to some extent straightforward. More specifically, for strictly polygonal-shaped domains, property (i) is a direct consequence of Theorem 1.1 in [18]. For curvilinear polygons, the proof of (i) is similar to the one used for establishing Proposition 4.9.

Next, we prove part (ii). We set:

$$
\mathbb{P}^{2}=\mathbb{P}^{1} \times \mathbb{P}^{1}
$$

where $\mathbb{P}^{1}$ is defined in Proposition 4.9. It follows from Proposition 4.9 and the tensor product's properties that:

$$
T^{2}=T^{1} \otimes T^{1}: W=\left(H^{2}(\Omega) \cap H_{0}^{1}(\Omega)\right)^{2} \longrightarrow \mathbb{P}^{2}
$$

is linear, continuous, and surjective. In addition, $T^{2}$ admits a linear and continuous right inverse, denoted by $R^{2}$, i.e., $T^{2} \circ R^{2}=I_{\mathbb{P}^{2}}$.

Let $u=\left(u_{1}, u_{2}\right) \in W$ and $f$ given by:

$$
f=T^{2}(u)
$$

Let $S$ be the mapping such that:

$$
\sigma(u) \nu_{\mid \Gamma_{j}}=S(u)_{j} ; j=1, \ldots, M .
$$

In the following, we prove that:

$$
S(u) \in \mathbb{P}^{2},
$$

and for $j=1, \ldots, M$, there is an invertible $2 \times 2$ matrix $A_{j}$ which satisfies the following three properties:

(a)

$$
S(u)_{j}=A_{j}\left(T^{2} u\right)_{j}
$$

(b)

$$
\operatorname{det} \mathrm{A}_{\mathrm{j}}=\mu(\lambda+2 \mu)
$$

that is the determinant of $A_{j}$ does not depend on the curvature of $\Gamma_{j}$.

(c) The entries of $A_{j}$ are Lipschitz continuous.

We first prove $\mathrm{Eq}$ (4.27). For $j=1, \ldots, M$, consider the line segment $\Gamma_{j}$. At any point $x \in \Gamma_{j}$, we denote by $\tau=\left(\tau_{1}, \tau_{2}\right)^{t}$ (resp. $\left.\nu=\left(\nu_{1}, \nu_{2}\right)^{t}\right)$ the unit tangent (normal) vector to $\Gamma_{j}$ (we omit here to use the subscript $j$ for simplicity purposes). Recall that the basis $\{\tau, \nu\}$ has the canonical orientation. Hence,

$$
\nu_{1}=-\tau_{2} \quad \text { and } \quad \nu_{2}=\tau_{1} .
$$


Using Eq (4.30), one can easily verify that for $u \in\left(H^{2}(\Omega) \cap H_{0}^{1}(\Omega)\right)^{2}$, we have:

$$
\left(\begin{array}{l}
\frac{\partial u}{\partial \tau} \\
\frac{\partial u}{\partial \nu}
\end{array}\right)=B\left(\begin{array}{l}
\frac{\partial u}{\partial x} \\
\frac{\partial u}{\partial y}
\end{array}\right),
$$

where $B$ is a $2 \times 2$ matrix given by:

$$
B=\left(\begin{array}{cc}
\nu_{2} & -\nu_{1} \\
\nu_{1} & \nu_{2}
\end{array}\right)
$$

Hence, $B$ is invertible (det $\mathrm{B}=1)$ and we have:

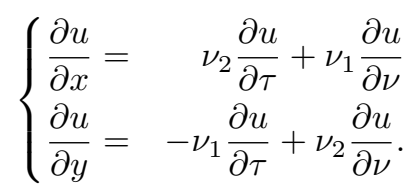

Since $u \in\left(H_{0}^{1}(\Omega)\right)^{2}$, it follows from Eq. (4.33) that:

$$
\left\{\begin{array}{l}
\frac{\partial u}{\partial x}=\nu_{1} \frac{\partial u}{\partial \nu} \\
\frac{\partial u}{\partial y}=\nu_{2} \frac{\partial u}{\partial \nu}
\end{array}\right.
$$

Therefore,

$$
\frac{\partial u}{\partial x}, \frac{\partial u}{\partial y} \in \tilde{\mathbf{H}}^{1 / 2}\left(\Gamma_{j}\right) ; j=1, \ldots, M
$$

Consequently,

$$
\varepsilon_{i j}, \sigma_{i j} \in \tilde{\mathbf{H}}^{1 / 2}\left(\Gamma_{j}\right) ; j=1, \ldots, M
$$

Next, we determine the matrix $A_{j}$. To this end, we express $\sigma(u) \nu$ in terms of $\frac{\partial u}{\partial \nu}$. For $j=1, \ldots, M$, we have:

$$
\sigma(u) \nu=A_{j} \frac{\partial u}{\partial \nu} \quad \text { on } \Gamma_{j}
$$

where

$$
A_{j}=\left(\begin{array}{cc}
\mu+(\lambda+\mu) \nu_{1}^{2} & (\lambda+\mu) \nu_{1} \nu_{2} \\
(\lambda+\mu) \nu_{1} \nu_{2} & \mu+(\lambda+\mu) \nu_{2}^{2}
\end{array}\right) .
$$

Since $\Gamma_{j}$ is $\mathcal{C}^{1,1}$, then $\nu_{1}$ and $\nu_{2}$ are Lipschitz continuous. Therefore, $\nu_{1}^{2}, \nu_{2}^{2}$ and $\nu_{1} \nu_{2}$ are also Lipschitz continuous on $\Gamma_{j}$.

Furthermore, since $\nu_{1}^{2}+\nu_{2}^{2}=1$, then it is easy to verify that:

$$
\operatorname{det} \mathrm{A}_{\mathrm{j}}=\mu(\lambda+2 \mu) ; \mathrm{j}=1, \ldots, \mathrm{M},
$$

that is the determinant of $A_{j}$ does not depend on the curvature of $\Gamma_{j}$. Hence, $A_{j}$ is an isomorphism on $\left(\tilde{H}^{1 / 2}\left(\Gamma_{j}\right)\right)^{2}$ which ensures the surjectivity of the mapping. 
Remark 4.11. Observe that we can characterize explicitly the right inverse of $T^{2}$. Indeed, we have:

$$
\mathcal{R}_{\sigma}=R^{2} \underset{j=1}{M} A_{j}^{-1}
$$

where, for $f^{j} \in\left(\tilde{H}^{1 / 2}\left(\Gamma_{j}\right)\right)^{2}$, we have:

$$
\left(\bigotimes_{j=1}^{M} A_{j}^{-1}\right)\left(f^{1}, \ldots, f^{M}\right)^{t}=\left(A_{1}^{-1} f^{1}, \ldots, A_{M}^{-1} f^{M}\right)^{t}
$$

and $R^{2}$ is the extension mapping of the normal derivatives given by the Corollary of Theorem 1.1 and Lemma 3.5 for $s=0$ in [18].

Next, we construct two lifting operators for the trace mappings $\gamma_{0, j}$ and $\gamma_{\sigma, j}$, respectively. These operators are needed to prove Proposition 4.13

Lemma 4.12. For $j=1, \ldots, M$, there are two positive constants $C_{0}$ and $C_{1}$, that depend on $\Gamma_{j}$ only, such that:

(i) For $\zeta \in \tilde{\mathbf{H}}^{3 / 2}\left(\Gamma_{j}\right)$, there is $\varphi \in \mathbf{H}_{j}^{N}\left(\Omega^{s}\right)$ such that $\gamma_{0, j}(\varphi)=\zeta$ and

$$
\|\varphi\|_{H^{2}\left(\Omega^{s}\right)} \leq C_{0}\|\zeta\|_{\tilde{\mathbf{H}}^{3 / 2}\left(\Gamma_{j}\right)} .
$$

(ii) For $\xi \in \tilde{\mathbf{H}}^{1 / 2}\left(\Gamma_{j}\right)$, there is $\psi \in \mathbf{H}_{j}^{D}\left(\Omega^{s}\right)$ such that $\gamma_{\sigma, j}(\psi)=\xi$ and

$$
\|\psi\|_{H^{2}\left(\Omega^{s}\right)} \leq C_{1}\|\xi\|_{\tilde{\mathbf{H}}^{1 / 2}\left(\Gamma_{j}\right)} .
$$

Proof. First, we prove part (i). Let $j \in\{1, \ldots, M\}$. We already know (see Proposition 4.10) that the mapping $\gamma_{0, j}: u \mapsto u_{\mid \Gamma_{j}}$ is linear, continuous, and surjective from $\mathbf{H}_{j}^{N}\left(\Omega^{s}\right)$ to $\tilde{\mathbf{H}}^{3 / 2}\left(\Gamma_{j}\right)$. In addition, its nullspace is $\left(H_{0}^{2}\left(\Omega^{s}\right)\right)^{2}$. Therefore, $\gamma_{0, j}$ is linear, continuous, and bijective from $\mathbf{H}_{j}^{N}\left(\Omega^{s}\right) /\left(H_{0}^{2}\left(\Omega^{s}\right)\right)^{2}$ into $\tilde{\mathbf{H}}^{3 / 2}\left(\Gamma_{j}\right)$. The open mapping theorem (see Theorem 6.6 in [41]) allows to conclude that $\gamma_{0, j}$ is an isomorphism from $\mathbf{H}_{j}^{N}\left(\Omega^{s}\right) /\left(H_{0}^{2}\left(\Omega^{s}\right)\right)^{2}$ to $\tilde{\mathbf{H}}^{3 / 2}\left(\Gamma_{j}\right)$. Therefore, there is a positive constant $C_{0}$, that depends on $\Gamma_{j}$ only, such that, for any $\zeta \in \tilde{\mathbf{H}}^{3 / 2}\left(\Gamma_{j}\right)$, there is $\varphi \in \mathbf{H}_{j}^{N}\left(\Omega^{s}\right)$ that satisfies:

$$
\|\varphi\|_{H^{2}\left(\Omega^{s}\right)} \leq C_{0}\|\zeta\|_{\tilde{\mathbf{H}}^{3 / 2}\left(\Gamma_{j}\right)},
$$

which concludes the proof of part (i).

Next, we prove property (ii) of Lemma 4.12. We already know (see Proposition 4.10) that, for $j \in\{1, \ldots, M\}$, the mapping $\gamma_{\sigma, j}: u \mapsto \sigma(u) \nu_{\mid \Gamma_{j}}$ is linear, continuous, and surjective from $\mathbf{H}_{j}^{D}\left(\Omega^{s}\right)$ into $\tilde{\mathbf{H}}^{1 / 2}\left(\Gamma_{j}\right)$. In addition, its nullspace is $\left(H_{0}^{2}\left(\Omega^{s}\right)\right)^{2}$. Therefore, $\gamma_{\sigma, j}$ is linear, continuous, and bijective from $\mathbf{H}_{j}^{D}\left(\Omega^{s}\right) /\left(H_{0}^{2}\left(\Omega^{s}\right)\right)^{2}$ into $\tilde{\mathbf{H}}^{1 / 2}\left(\Gamma_{j}\right)$. It follows from the open mapping theorem (see Theorem 6.6 in [41]) that the trace of the traction field is an isomorphism from $\left.\tilde{\mathbf{H}}^{1 / 2}\left(\Gamma_{j}\right)\right) /\left(H_{0}^{2}\left(\Omega^{s}\right)\right)^{2}$ to $\tilde{\mathbf{H}}^{1 / 2}\left(\Gamma_{j}\right)$. Hence, there is a positive constant $C_{1}$, that depends on $\Gamma_{j}$ only, such that, for any $\xi \in \tilde{\mathbf{H}}^{1 / 2}\left(\Gamma_{j}\right)$, there is $\varphi \in \mathbf{H}_{j}^{D}\left(\Omega^{s}\right)$ which satisfies:

$$
\|\psi\|_{H^{2}\left(\Omega^{s}\right)} \leq C_{1}\|\xi\|_{\tilde{\mathbf{H}}^{1 / 2}\left(\Gamma_{j}\right)} .
$$


The following results formulated as a proposition extend the trace properties stated in Proposition 4.10 to the space $D\left(\nabla \cdot \sigma, \Omega^{s}\right)$. This proposition provides key results for proving that the expressions given by Eqs. (3.7)-(3.8) are well-defined. We must point out that, since the boundary $\Gamma$ is assumed to be a Lipschitz continuous polygonalshaped boundary, it is not possible to prove Proposition 4.13 by using the standard approach based on the duality and the density arguments [34, 19, 20].

Proposition 4.13. For $j=1, \ldots, M$, we have:

(i) The mapping $\gamma_{0, j}: u \mapsto u_{\mid \Gamma_{j}}$ is linear and continuous from $D\left(\nabla \cdot \sigma, \Omega^{s}\right)$ to $\tilde{\mathbf{H}}^{-1 / 2}\left(\Gamma_{j}\right)$.

(ii) The mapping $\gamma_{\sigma, j}: u \mapsto \sigma(u) \nu_{\mid \Gamma_{j}}$ is linear and continuous from $D\left(\nabla \cdot \sigma, \Omega^{s}\right)$ to $\tilde{\mathbf{H}}^{-3 / 2}\left(\Gamma_{j}\right)$.

(ii) For $u \in D\left(\nabla \cdot \sigma, \Omega^{s}\right)$, we have:

$$
\int_{\Omega^{s}} u \nabla \cdot \sigma(\varphi) d x-\int_{\Omega^{s}} \nabla \cdot \sigma(u) \varphi d x=\left\langle u, \sigma(\varphi) \nu_{j}\right\rangle_{\sim,-1 / 2,1 / 2, \Gamma_{j}} ; \forall \varphi \in \mathbf{H}_{j}^{D}\left(\Omega^{s}\right) .
$$

$$
\int_{\Omega^{s}} u \nabla \cdot \sigma(\psi) d x-\int_{\Omega^{s}} \nabla \cdot \sigma(u) \psi d x=-\left\langle\sigma(u) \nu_{j}, \psi\right\rangle_{\sim,-3 / 2,3 / 2, \Gamma_{j}} ; \forall \psi \in \mathbf{H}_{j}^{N}\left(\Omega^{s}\right)
$$

Proof. We use the lifting operators introduced in Lemma 4.12 along with the density results stated in Lemma 4.7 to establish Proposition 4.13. Note that our approach in the proposed proof follows the ideas in $[2,3,4]$ to establish similar results in the case of the Laplace operator.

First, we prove the result for the trace operator $\gamma_{0, j}$ given in part (i) of Proposition 4.13. Let $(u, v)$ be in $\left(H^{2}\left(\Omega^{s}\right)\right)^{2} \times \mathbf{H}_{j}^{D}\left(\Omega^{s}\right)$. It follows from Green-Riemann formula that:

$$
\begin{aligned}
\int_{\Gamma_{j}} u \cdot \sigma(v) \nu d s & =\int_{\Omega^{s}} u \cdot(\nabla \cdot \sigma(v)) d x+\int_{\Omega^{s}} \nabla u: \nabla v d x \\
& =\int_{\Omega^{s}} u \cdot(\nabla \cdot \sigma(v)) d x-\int_{\Omega^{s}}(\nabla \cdot \sigma(u)) \cdot v d x+\int_{\Gamma} \sigma(u) \nu \cdot v d s \\
& =\int_{\Omega^{s}} u \cdot(\nabla \cdot \sigma(v)) d x-\int_{\Omega^{s}}(\nabla \cdot \sigma(u)) \cdot v d x
\end{aligned}
$$

Therefore, we have:

$$
\begin{aligned}
\left|\int_{\Gamma_{j}} u \cdot \sigma(v) \nu d s\right| & \leq\|u\|_{\nabla \cdot \sigma}\|v\|_{\nabla \cdot \sigma} \\
& \leq\|u\|_{\nabla \cdot \sigma}\|v\|_{\left(H^{2}\left(\Omega^{s}\right)\right)^{2}}
\end{aligned}
$$

Using the lifting operator introduced in Lemma 4.12 (see property (ii)), we conclude that there is a positive constant $C_{1}$ (that depends on $\Gamma_{j}$ only) such that, for $\xi=$ $\gamma_{\sigma, j}(v) \in \tilde{\mathbf{H}}^{1 / 2}\left(\Gamma_{j}\right):$

$$
\left|\int_{\Gamma_{j}} u \xi d s\right| \leq C_{1}\|v\|_{\nabla \cdot \sigma}\|\xi\|_{\tilde{\mathbf{H}}^{1 / 2}\left(\Gamma_{j}\right)}
$$


Recall that the norm of the dual space $\tilde{\mathbf{H}}^{-1 / 2}\left(\Gamma_{j}\right)$ of $\tilde{\mathbf{H}}^{1 / 2}\left(\Gamma_{j}\right)$ is given by:

$$
\|\psi\|_{\tilde{\mathbf{H}}^{-1 / 2}\left(\Gamma_{j}\right)}=\sup _{\xi \in \tilde{\mathbf{H}}^{1 / 2}\left(\Gamma_{j}\right),\|\xi\|_{\tilde{\mathbf{H}}^{1 / 2}\left(\Gamma_{j}\right)}=1}\langle\psi, \xi\rangle_{\sim,-1 / 2,1 / 2, \Gamma_{j}} .
$$

Hence, we have:

$$
\|u\|_{\tilde{\mathbf{H}}^{-1 / 2}\left(\Gamma_{j}\right)} \leq C_{1}\|u\|_{\nabla \cdot \sigma} .
$$

Consequently, the linear operator $\gamma_{0, j}$ is continuous from $\left(H^{2}\left(\Omega^{s}\right)\right)^{2}$, equipped with the norm $\|\cdot\|_{\nabla \cdot \sigma}$ into $\tilde{\mathbf{H}}^{-1 / 2}\left(\Gamma_{j}\right)$.

Moreover, $\left(H^{2}\left(\Omega^{s}\right)\right)^{2}$ is dense in $D\left(\nabla \cdot \sigma, \Omega^{s}\right)$ (see Lemma 4.7). Therefore, we can use Hahn-Banach theorem (see Chapter IV, Section 5 in [41]) when applied to the extension of linear and continuous forms on a subspace [39]. We conclude that the trace mapping $\gamma_{0, j}$ can be extended by continuity to a linear continuous operator from $D\left(\nabla \cdot \sigma, \Omega^{s}\right)$ into $\tilde{\mathbf{H}}^{-1 / 2}\left(\Gamma_{j}\right)$.

Second, we prove the result for the traction field operator. Let $(u, v)$ be in $\left(H^{2}\left(\Omega^{s}\right)\right)^{2} \times$ $\mathbf{H}_{j}^{N}\left(\Omega^{s}\right)$. We apply the standard integration by parts formula and obtain:

$$
\begin{aligned}
\int_{\Gamma_{j}} \sigma(u) \nu \cdot v d s & =\int_{\Omega^{s}}(\nabla \cdot \sigma(u)) \cdot v d x+\int_{\Omega^{s}} \nabla u: \nabla v d x \\
& =\int_{\Omega^{s}}(\nabla \cdot \sigma(u)) \cdot v d x-\int_{\Omega^{s}} u \cdot(\nabla \cdot \sigma(v)) d x+\int_{\Gamma} u \cdot \sigma(v) \nu d s \\
& =\int_{\Omega^{s}}(\nabla \cdot \sigma(u)) \cdot v d x-\int_{\Omega^{s}} u \cdot(\nabla \cdot \sigma(v)) d x .
\end{aligned}
$$

It follows that:

$$
\begin{aligned}
\left|\int_{\Gamma_{j}} \sigma(u) \nu \cdot v d s\right| & \leq\|u\|_{\nabla \cdot \sigma}\|v\|_{\nabla \cdot \sigma} \\
& \leq\|u\|_{\nabla \cdot \sigma}\|v\|_{\left(H^{2}\left(\Omega^{s}\right)\right)^{2}}
\end{aligned}
$$

We can then use the lifting operator introduced in Lemma 4.12 (see property (i)) for the trace operator $\gamma_{0, j}$. Hence, there is a positive constant $C_{0}$ (that depends on $\Gamma_{j}$ only) such that, for $\zeta=\gamma_{0, j}(v) \in \tilde{\mathbf{H}}^{3 / 2}\left(\Gamma_{j}\right)$, we have:

$$
\left|\int_{\Gamma_{j}} \sigma(u) \nu \cdot \zeta d s\right| \leq C_{0}\|u\|_{\nabla \cdot \sigma}\|\zeta\|_{\tilde{\mathbf{H}}^{3 / 2}\left(\Gamma_{j}\right)} .
$$

Since the dual space $\tilde{\mathbf{H}}^{-3 / 2}\left(\Gamma_{j}\right)$ is equipped with the following dual norm:

$$
\|\varphi\|_{\tilde{\mathbf{H}}^{-3 / 2}\left(\Gamma_{j}\right)}=\sup _{\zeta \in \tilde{\mathbf{H}}^{3 / 2}\left(\Gamma_{j}\right),\|\zeta\|_{\tilde{\mathbf{H}}^{3 / 2}\left(\Gamma_{j}\right)}=1}\langle\varphi, \zeta\rangle_{\sim,-3 / 2,3 / 2, \Gamma_{j}},
$$

we deduce that:

$$
\|\sigma(u) \nu\|_{\tilde{\mathbf{H}}^{-3 / 2}\left(\Gamma_{j}\right)} \leq C_{0}\|u\|_{\nabla \cdot \sigma} .
$$

We conclude that the linear operator $\gamma_{\sigma, j}$ is continuous from $\left(\left(H^{2}\left(\Omega^{s}\right)\right)^{2},\|\cdot\|_{\nabla \cdot \sigma}\right)$ into $\left(\tilde{\mathbf{H}}^{-3 / 2}\left(\Gamma_{j}\right),\|\cdot\|_{\tilde{\mathbf{H}}^{-3 / 2}\left(\Gamma_{j}\right)}\right)$. Finally, since $\left(H^{2}\left(\Omega^{s}\right)\right)^{2}$ is dense in $D\left(\nabla \cdot \sigma, \Omega^{s}\right)$ (see 
Lemma 4.7), we can apply the Hahn-Banach theorem (see Chapter IV, Section 5 in [41]). Hence, the mapping $\gamma_{\sigma, j}$ can thus be extended to a linear and continuous operator from $D\left(\nabla \cdot \sigma, \Omega^{s}\right)$ into $\tilde{\mathbf{H}}^{-3 / 2}\left(\Gamma_{j}\right)$.

The Green formulae given by (iii) are then easily derived as immediate consequences of (i) and (ii).

5. Proof of Theorem 3.2. This section is devoted to the proof of Theorem 3.2. To this end, we first establish that $u^{\prime}$ satisfies the first equation of BVP (2) and $p^{\prime}$ satisfies the second and last equations of BVP (2).

Lemma 5.14. Let $\left(p^{\prime}, u^{\prime}\right)$ be the local derivative of the elasto-acoustic field $(p, u)$ at $\theta=0$ in the direction $h \in\left(\mathcal{C}^{2}\left(\mathbb{R}^{2}\right)\right)^{2}$. Then, $\left(p^{\prime}, u^{\prime}\right)$ satisfies the following:

$$
\begin{aligned}
\Delta p^{\prime}+k^{2} p^{\prime}=0 & \text { in } \Omega^{f}, \\
\nabla \cdot \sigma\left(u^{\prime}\right)+\omega^{2} \rho_{s} u^{\prime}=0 & \text { in } \Omega^{s},
\end{aligned}
$$

and

$$
\lim _{r \rightarrow+\infty} \sqrt{r}\left(\frac{\partial p^{\prime}}{\partial r}-\mathrm{i} \frac{\omega}{\rho_{f}} p^{\prime}\right)=0 .
$$

Proof. We only establish Eq. (5.38) since the proofs for Eq. (5.37) and Eq. (5.39) can be found in reference [12] where only the exterior Helmholtz problem was considered and assuming $\Gamma$ to be Lipschitz continuous. We set:

$$
B=\nabla \cdot \sigma+\omega^{2} \rho_{s} I
$$

and

$$
\phi_{\theta}=B u_{\theta}
$$

Note that:

$$
\phi_{0}=B u,
$$

where $u$ is the solution of the elasto-acoustic scattering problem BVP (1) for $\theta=0$. Since the elastic scattered field $u_{\theta}$ satisfies the elastodynamics equation given by Eq. (a) in BVP (1), we have:

$$
\phi_{\theta}=0 \quad \text { in } \Omega_{\theta}^{s},
$$

which can be re-written in the reference domain $\Omega^{s}$ as follows:

$$
\phi_{\theta} \circ(I+\theta)=0 \quad \text { in } \Omega^{s} .
$$

On the other hand, $B$ is a linear and continuous operator from $\left(H^{1}\left(\Omega^{s}\right)\right)^{2}$ into $\left(\mathcal{D}^{\prime}\left(\Omega^{s}\right)\right)^{2}$. Hence, $B$ is differentiable -at least- in the distribution sense, that is, $v \mapsto\langle B v, \varphi\rangle$ is differentiable for each $\varphi \in \mathcal{D}\left(\Omega^{s}\right)$, and we have:

$$
\frac{\partial B}{\partial v}=B .
$$

Furthermore, the mappings $\theta \mapsto u_{\theta} \circ(I+\theta)$ and $\theta \mapsto u_{\theta}$ are differentiable [7, 14]. Therefore, $\theta \mapsto \phi_{\theta} \circ(I+\theta)$ and $\theta \mapsto \phi_{\theta}$ are continuously Fréchet differentiable, 
and locally continuously Fréchet differentiable -at least- in the distribution sense at $\theta=0$ in the direction $h \in\left(\mathcal{C}^{2}\left(\mathbb{R}^{2}\right)\right)^{2}$. Consequently, for an admissible perturbation $\theta \in\left(\mathcal{C}^{1}\left(\mathbb{R}^{2}\right)\right)^{2}$ in a neighborhood of zero, we obtain:

$$
\frac{\partial \phi_{\theta}}{\partial \theta}(0) h=\frac{\partial}{\partial \theta}\left(\phi_{\theta} \circ(I+\theta)\right)(0) h-\nabla \phi(0) h \quad \text { in } \Omega^{s} .
$$

In addition, using Eq. (5.42), we obtain:

$$
\nabla \phi(0)=\nabla(B u)=0 \text { in } \Omega^{s} .
$$

Moreover, we deduce from substituting Eq. (5.44) into Eq. (5.46) that:

$$
\frac{\partial \phi_{\theta}}{\partial \theta}(0) h=\frac{\partial B}{\partial u} \frac{\partial u_{\theta}}{\partial \theta}(0) h=0 \quad \text { in } \Omega^{s} .
$$

Finally, we deduce from Eq. (5.40), Eq. (5.45) and Eq. (5.48) that:

$$
\nabla \cdot \sigma\left(u^{\prime}\right)+\omega^{2} \rho_{s} u^{\prime}=0 \quad \text { in } \Omega^{s},
$$

which proves Eq. (5.38).

To establish the boundary conditions stated in Theorem 3.2, we proceed in two steps. In Step 1, we derive these conditions assuming the boundary $\Gamma$ to be regular enough $\left(\mathcal{C}^{k}, k \geq 2\right)$. In Step 2, we prove that the obtained transmission conditions are well-defined when $\Gamma$ is assumed to be a polygonal-shaped boundary of class Lipschitz continuous $\left(\mathcal{C}^{0,1}\right)$.

5.1. Derivation of the transmission conditions for regular boundaries. We assume in this section that the boundary $\Gamma$ is regular enough $\left(\mathcal{C}^{k}, k \geq 2\right)$.

The following result pertains to the derivation of the first transmission condition (see Eq. (c) in BVP (2)).

Proposition 5.15. Let $(u, p)$ be the solution of BVP (1) and $\left(u^{\prime}, p^{\prime}\right)$ its corresponding local derivative at $\theta=0$ in the direction $h \in\left(\mathcal{C}^{2}\left(\mathbb{R}^{2}\right)\right)^{2}$. Then, $\left(u^{\prime}, p^{\prime}\right)$ satisfies the following boundary equation:

$$
\tau\left(u^{\prime}\right)=-p^{\prime} \nu+F(u, p, h) \quad \text { on } \Gamma \text { a.e. }
$$

where $F$ is given by Eq. (3.7).

Proof. Let $\theta$ be an admissible perturbation in $\left(\mathcal{C}^{1}\left(\mathbb{R}^{2}\right)\right)^{2}$. Then, it follows from Eq. (c) of BVP (1) that:

$$
\left(\sigma\left(u_{\theta}\right)+p_{\theta}+g\right) \nu_{\theta}=0 \quad \text { on } \Gamma_{\theta} \quad \text { a.e. . }
$$

We set:

$$
\phi_{\theta}=\sigma\left(u_{\theta}\right)+p_{\theta}+g .
$$

Hence, it follows from Eq. (5.51) and Eq. (5.52) that:

$$
\phi_{\theta} \nu_{\theta}=0 \quad \text { on } \Gamma_{\theta} \text { a.e. }
$$

which can be re-written on the reference boundary $\Gamma$ as follows:

$$
\phi_{\theta} \circ(I+\theta) \nu_{\theta} \circ(I+\theta)=0 \quad \text { on } \Gamma \quad \text { a.e. . }
$$


Furthermore (see [33], Lemma 4.8), we have:

$$
\nu_{\theta} \circ(I+\theta)=\frac{1}{\|J(\theta) \nu\|_{2}} J(\theta) \nu \quad \text { on } \Gamma \quad \text { a.e. },
$$

where

$$
J(\theta)=\left[(I+\theta)^{\prime}\right]^{-t} \quad \text { in } \mathbb{R}^{2} .
$$

Observe that:

$$
J(0)=I .
$$

Substituting Eq. (5.55) and Eq. (5.56) into Eq. (5.54) leads to:

$$
\phi_{\theta} \circ(I+\theta) J(\theta) \nu=0 \quad \text { on } \Gamma \text { a.e. . }
$$

Next, we evaluate the Fréchet derivative of Eq. (5.58) with respect to $\theta$. To this end, we extend Eq. (5.58) to an open set $D$ containing $\Gamma_{\theta}$. Let the extension of the unit normal vector $\nu$ into $\left(L^{\infty}\left(\mathbb{R}^{2}\right)\right)^{2}$ be still denoted by $\nu$. Likewise, the transported solution $\left(u_{\theta} \circ(I+\theta), p_{\theta} \circ(I+\theta)\right)$ is extended into the whole $D$ and the extension is also denoted by $\left(u_{\theta} \circ(I+\theta), p_{\theta} \circ(I+\theta)\right)$.

We consider the following auxiliary function $\varphi_{\theta}$ defined in the domain $D$ by:

$$
\varphi_{\theta}=\phi_{\theta} \circ(I+\theta) J(\theta) \nu \quad \text { in } D \quad \text { a.e. . }
$$

Note that $\varphi_{\theta}$ satisfies:

$$
\varphi_{\theta}=0 \quad \text { on } \Gamma \text { a.e. . }
$$

Observe that the mappings $\theta \mapsto p_{\theta} \circ(I+\theta)$ and $\theta \mapsto u_{\theta} \circ(I+\theta)$ are differentiable at $\theta=0$ in the direction $h \in\left(\mathcal{C}^{2}\left(\mathbb{R}^{2}\right)\right)^{2}[7,14]$. In addition, since (a) $\theta \mapsto g \circ(I+\theta)$ is differentiable, and (b) the stress tensor operator $\sigma$ is linear and continuous, then it follows that $\theta \mapsto \phi_{\theta}$ is differentiable -at least- in the distribution sense at $\theta=0$ in the direction $h \in\left(\mathcal{C}^{2}\left(\mathbb{R}^{2}\right)\right)^{2}$.

Furthermore, the mapping $\theta \mapsto J(\theta)$ is differentiable at $\theta=0$ in the direction $h \in$ $\left(\mathcal{C}^{2}\left(\mathbb{R}^{2}\right)\right)^{2}$ from $\left(\mathcal{C}^{1}\left(\mathbb{R}^{2}\right)\right)^{2}$ into $\left(\mathcal{C}^{1}\left(\mathbb{R}^{2}\right)\right)^{2}$, and we have (see Eq.(44) in [12]),

$$
\frac{\partial J(\theta)}{\partial \theta}(0) h=-\left[h^{\prime}\right]^{t} .
$$

Consequently, the mapping $\theta \mapsto \varphi_{\theta}$ is differentiable -at least- in the distribution sense at $\theta=0$ in the direction $h \in\left(\mathcal{C}^{2}\left(\mathbb{R}^{2}\right)\right)^{2}$. Next, we evaluate its local derivative with respect to $\theta$. We proceed by evaluating the derivative of each term of $\varphi_{\theta}$. We have:

$$
\begin{aligned}
\frac{\partial}{\partial \theta}\left(p_{\theta} \circ(I+\theta) J(\theta) \nu\right)(0) h= & \frac{\partial}{\partial \theta}\left(p_{\theta} \circ(I+\theta)\right)(0) h J(0) \nu \\
& +p_{\theta} \circ(I+\theta)_{\mid \theta=0} \frac{\partial J(\theta)}{\partial \theta}(0) h \nu .
\end{aligned}
$$

Moreover, it follows from applying the chain rule that:

$$
\frac{\partial}{\partial \theta}\left(p_{\theta} \circ(I+\theta)\right)(0) h=\frac{\partial p_{\theta}}{\partial \theta}(0) h+\nabla p_{\theta}(0) \cdot h .
$$


Consequently, it follows from substituting Eqs. (5.63), (5.61) and (5.57) into Eq. (5.62) that:

$$
\frac{\partial}{\partial \theta}\left(p_{\theta} \circ(I+\theta) J(\theta) \nu\right)(0) h=\left(\frac{\partial p_{\theta}}{\partial \theta}(0) h+\nabla p_{\theta}(0) \cdot h\right) \nu-p\left[h^{\prime}\right]^{t} \nu,
$$

that is:

$$
\frac{\partial}{\partial \theta}\left(p_{\theta} \circ(I+\theta) J(\theta) \nu\right)(0) h=\left(p^{\prime}+\nabla p \cdot h\right) \nu-p\left[h^{\prime}\right]^{t} \nu .
$$

Similarly, we have:

$$
\frac{\partial}{\partial \theta}(g \circ(I+\theta) J(\theta) \nu)(0) h=\nabla g \cdot h \nu-g\left[h^{\prime}\right]^{t} \nu .
$$

On the other hand, the computation of $\frac{\partial}{\partial \theta}\left(\sigma\left(u_{\theta}\right) \circ(I+\theta) J(\theta) \nu\right)(0) h$ deserves a special attention due to the presence of the stress tensor $\sigma$. Indeed, we evaluate this local derivative by considering each tensor coordinate. More specifically, let $\sigma_{l}\left(u_{\theta}\right)$ be the $l^{\text {th }}$ row vector of the matrix $\sigma\left(u_{\theta}\right), l=1,2$. Then, we have:

$$
\frac{\partial}{\partial \theta}\left(\sigma\left(u_{\theta}\right) \circ(I+\theta) J(\theta) \nu\right)(0) h=\left[\frac{\partial}{\partial \theta}\left(\sigma_{l}\left(u_{\theta}\right) \circ(I+\theta) J(\theta) \nu\right)(0) h\right]_{1 \leq l \leq 2} .
$$

On the other hand, we have:

$$
\begin{aligned}
& \frac{\partial}{\partial \theta}\left(\sigma_{l}\left(u_{\theta}\right) \circ(I+\theta) J(\theta) \nu\right)(0) h \\
& =\frac{\partial}{\partial \theta}\left(\sigma_{l}\left(u_{\theta}\right) \circ(I+\theta)\right)(0) h J(0) \nu+\sigma_{l}\left(u_{\theta}\right) \circ(I+\theta)_{\left.\right|_{\theta=0}} \frac{\partial J(\theta)}{\partial \theta}(0) h \nu ; \quad l=1,2 .
\end{aligned}
$$

Moreover, applying the chain rule to each entry $\sigma_{l m}(u)$ of the row vector $\sigma_{l}(u)$, as in Eq. (5.63), leads to:

$$
\begin{aligned}
& \frac{\partial}{\partial \theta}\left(\sigma_{l}\left(u_{\theta}\right) \circ(I+\theta)\right)(0) h \\
& =\left[\frac{\partial}{\partial \theta}\left(\sigma_{l m}\left(u_{\theta}\right) \circ(I+\theta)\right)(0) h\right]_{1 \leq m \leq 2} \\
& =\left[\frac{\partial \sigma_{l m}\left(u_{\theta}\right)}{\partial \theta}(0) h+h \cdot \nabla \sigma_{l m}\left(u_{\theta}\right)(0)\right]_{1 \leq m \leq 2} \\
& =\left[\sigma_{l m}\left(\frac{\partial u_{\theta}}{\partial \theta}(0) h\right)+h^{t} \nabla \sigma_{l m}(u)\right]_{1 \leq m \leq 2} .
\end{aligned}
$$

Let $\nabla \sigma_{l}(u)$ be the Jacobian matrix defined by:

$$
\nabla \sigma_{l}(u)=\left[\nabla \sigma_{l}(u)^{j m}\right]_{1 \leq j, m \leq 2}=\left[\frac{\partial \sigma_{l m}(u)}{\partial x_{j}}\right]_{1 \leq j, m \leq 2} .
$$

Thus, we have:

$$
\begin{aligned}
& \frac{\partial}{\partial \theta}\left(\sigma_{l}\left(u_{\theta}\right) \circ(I+\theta) J(\theta) \nu\right)(0) h \\
& =\left(\sigma_{l}\left(\frac{\partial u_{\theta}}{\partial \theta}(0) h\right)+h^{t} \nabla \sigma_{l}(u)\right) \nu-\sigma_{l}(u)\left[h^{\prime}\right]^{t} \nu
\end{aligned}
$$


Consequently, we obtain:

$$
\frac{\partial}{\partial \theta}\left(\sigma_{l}\left(u_{\theta}\right) \circ(I+\theta) J(\theta) \nu\right)(0) h=\left(\sigma_{l}\left(u^{\prime}\right)+h^{t} \nabla \sigma_{l}(u)\right) \nu-\sigma_{l}(u)\left[h^{\prime}\right]^{t} \nu ; \quad 1 \leq l \leq 2 .
$$

Using the notation:

$$
h^{t} \nabla \sigma(u):=\left[h^{t} \nabla \sigma_{l}(u)\right]_{1 \leq l \leq 2},
$$

we deduce that:

$$
\frac{\partial}{\partial \theta}\left(\sigma\left(u_{\theta}\right) \circ(I+\theta) J(\theta) \nu\right)(0) h=\left(\sigma\left(u^{\prime}\right)+h^{t} \nabla \sigma(u)\right) \nu-\sigma(u)\left[h^{\prime}\right]^{t} \nu .
$$

Note that:

$$
h^{t} \nabla \sigma(u) \nu=\left[\sum_{m=1}^{2} h^{t} \nabla \sigma_{l m}(u) \nu_{m}\right]_{1 \leq l \leq 2} .
$$

Therefore, it follows from Eqs. (5.60), (5.64), (5.65), (5.68), and (5.69) that:

$$
\begin{aligned}
\frac{\partial \varphi_{\theta}}{\partial \theta}(0) h= & \sigma\left(u^{\prime}\right) \nu+h^{t} \nabla \sigma(u) \nu-\sigma(u)\left[h^{\prime}\right]^{t} \nu+ \\
& p^{\prime} \nu+\nabla p \cdot h \nu-p\left[h^{\prime}\right]^{t} \nu+\nabla g \cdot h \nu-g\left[h^{\prime}\right]^{t} \nu \text { in } D .
\end{aligned}
$$

Since the boundary $\Gamma$ is assumed to be regular enough $\left(\mathcal{C}^{k}, k \geq 2\right)$, each term in Eq. (5.70) is well-defined on $\Gamma$. Then, it follows from both equations (5.59) and (5.58) that:

$$
\begin{gathered}
\sigma\left(u^{\prime}\right) \nu+h^{t} \nabla \sigma(u) \nu-\sigma(u)\left[h^{\prime}\right]^{t} \nu+ \\
p^{\prime} \nu+\nabla p \cdot h \nu-p\left[h^{\prime}\right]^{t} \nu+\nabla g \cdot h \nu-g\left[h^{\prime}\right]^{t} \nu=0 \text { on } \Gamma .
\end{gathered}
$$

Eq. (5.71) can be re-written as follows:

$$
\tau\left(u^{\prime}\right)=-p^{\prime} \nu-h^{t} \nabla \sigma(u) \nu+\sigma(u)\left[h^{\prime}\right]^{t} \nu-\nabla p^{T} \cdot h \nu+p^{T}\left[h^{\prime}\right]^{t} \nu,
$$

which concludes the proof of Proposition 5.15.

Next, we derive the second transmission condition given by Eq. (d) in BVP (2). The result is stated in the following proposition.

Proposition 5.16. Let $(u, p)$ be the solution of BVP (1) and $\left(u^{\prime}, p^{\prime}\right)$ its corresponding local derivative at $\theta=0$ in the direction $h \in\left(\mathcal{C}^{2}\left(\mathbb{R}^{2}\right)\right)^{2}$. Then, $\left(u^{\prime}, p^{\prime}\right)$ satisfies the following boundary equation:

$$
\omega^{2} \rho_{f} u^{\prime} \cdot \nu=\frac{\partial p^{\prime}}{\partial \nu}+G(u, p, h) \quad \text { on } \Gamma \quad \text { a.e., }
$$

where $G$ is given by Eq. (3.8).

Proof. Let $\theta$ be an admissible perturbation in $\left(\mathcal{C}^{1}\left(\mathbb{R}^{2}\right)\right)^{2}$. We set:

$$
\psi_{\theta}=\omega^{2} \rho_{f} u_{\theta}-\nabla p_{\theta}-\nabla g .
$$

Then, it follows from Eq. (d) in BVP (1) that we have:

$$
\psi_{\theta} \cdot \nu_{\theta}=0 \quad \text { on } \Gamma_{\theta} \text { a.e. . }
$$


We re-write Eq. (5.75) on the reference boundary $\Gamma$ and obtain:

$$
\psi_{\theta} \circ(I+\theta) \cdot \nu_{\theta} \circ(I+\theta)=0 \quad \text { on } \Gamma \text { a.e. . }
$$

Eqs. (5.76) and (5.55) allow to conclude that:

$$
\psi_{\theta} \circ(I+\theta) \cdot J(\theta) \nu=0 \quad \text { on } \Gamma \quad \text { a.e. . }
$$

Next, we extend Eq. (5.77) to an open set $D$ containing $\Gamma_{\theta}$. Similarly to the proof of Proposition 5.15, $\nu$ denotes an extension of the unit normal vector $\nu$ into $\left(L^{\infty}\left(\mathbb{R}^{2}\right)\right)^{2}$, and the transported solution in the whole $D$ is also denoted by $\left(p_{\theta} \circ(I+\theta), u_{\theta} \circ(I+\theta)\right)$. We are then able to introduce the auxiliary function $\Psi_{\theta}$ defined on $D$ as follows:

$$
\Psi_{\theta}=\psi_{\theta} \circ(I+\theta) \cdot J(\theta) \nu \quad \text { in } D
$$

Observe that:

$$
\Psi_{\theta}=0 \quad \text { on } \Gamma \text { a.e. }
$$

Furthermore, the mappings $\theta \mapsto p_{\theta} \circ(I+\theta)$ and $\theta \mapsto u_{\theta} \circ(I+\theta)$ are differentiable at $\theta=0[7,14]$. Since $\theta \mapsto g \circ(I+\theta)$ is also differentiable and $\nabla$ is a linear and continuous operator, it follows that $\theta \mapsto \psi_{\theta} \circ(I+\theta)$ is differentiable - at least- in the distribution sense at $\theta=0$ in the direction $h \in\left(\mathcal{C}^{2}\left(\mathbb{R}^{2}\right)\right)^{2}$.

Let $\psi_{\theta}^{l}$ denote the $l^{t h}$ coordinate of the column vector $\psi_{\theta}$, where $l=1,2$. Then, it follows from applying the chain rule to a scalar function that [33]:

$$
\begin{aligned}
& \frac{\partial}{\partial \theta}\left(\psi_{\theta} \circ(I+\theta)\right)(0) h \\
& =\left[\frac{\partial}{\partial \theta}\left(\psi_{\theta}^{l} \circ(I+\theta)\right)(0) h\right]_{1 \leq l \leq 2} \\
& =\left[\frac{\partial \psi_{\theta}^{l}}{\partial \theta}(0) h+\nabla \psi_{\theta}^{l}(0) h\right]_{1 \leq l \leq 2} \\
& =\frac{\partial \psi_{\theta}}{\partial \theta}(0) h+\nabla \psi_{\theta}(0) h
\end{aligned}
$$

We are now ready to differentiate the function $\Psi_{\theta}$ given by Eq. (5.78) with respect to $\theta$. Using the chain rule and Eq. (5.61), we have:

$$
\begin{aligned}
\frac{\partial \Psi_{\theta}}{\partial \theta}(0) h= & \frac{\partial}{\partial \theta}\left(\psi_{\theta} \circ(I+\theta)\right)(0) h \cdot J(\theta)_{\left.\right|_{\theta=0}} \nu+ \\
& \psi_{\theta} \circ(I+\theta)_{\left.\right|_{\theta=0}} \cdot \frac{\partial J(\theta)}{\partial \theta}(0) h \nu \text { in } D .
\end{aligned}
$$

It follows from substituting Eq. (5.61) and Eq. (5.80) into Eq. (5.81) that:

$$
\frac{\partial \Psi_{\theta}}{\partial \theta}(0) h=\left(\frac{\partial \psi_{\theta}}{\partial \theta}(0) h+\nabla \psi_{\theta}(0) h\right) \cdot J(0) \nu-\psi_{\theta}(0) \cdot\left[h^{\prime}\right]^{t} \nu \text { in } D
$$

Using Eq. (5.57) and Eq. (5.74), we obtain that:

$$
\nabla \psi_{\theta}(0)=\omega^{2} \rho_{f} \nabla u-\nabla(\nabla p)-\nabla(\nabla g)
$$


where the gradient of the vector field $u$ is given by:

$$
\nabla u=\left[\nabla u_{l j}\right]_{1 \leq j, l \leq 2}=\left[\frac{\partial u_{l}}{\partial x_{j}}\right]_{1 \leq j, l \leq 2} .
$$

Furthermore, we also have:

$$
\frac{\partial \psi_{\theta}}{\partial \theta}(0) h=\omega^{2} \rho_{f} \frac{\partial u_{\theta}}{\partial \theta}(0) h-\nabla\left(\frac{\partial p_{\theta}}{\partial \theta}(0) h\right)-\nabla\left(\frac{\partial g}{\partial \theta}(0) h\right) .
$$

Hence,

$$
\frac{\partial \psi_{\theta}}{\partial \theta}(0) h=\omega^{2} \rho_{f} u^{\prime}-\nabla p^{\prime}
$$

From Eq. (5.82) to Eq. (5.85), we deduce that:

$$
\begin{aligned}
\frac{\partial \Psi_{\theta}}{\partial \theta}(0) h= & \left(\omega^{2} \rho_{f} u^{\prime}-\nabla p^{\prime}\right) \cdot \nu+\omega^{2} \rho_{f} \nabla u h \cdot \nu- \\
& \nabla(\nabla p) h \cdot \nu-\nabla(\nabla g) h \cdot \nu- \\
& \left(\omega^{2} \rho_{f} u-\nabla p-\nabla g\right) \cdot\left[h^{\prime}\right]^{t} \nu \quad \text { in } D .
\end{aligned}
$$

Since $\Gamma$ is $\mathcal{C}^{k}, k \geq 2$, then we obtain from Eq. (5.79) and Eq. (5.86) that:

$$
\begin{aligned}
& \left(\omega^{2} \rho_{f} u^{\prime}-\nabla p^{\prime}\right) \cdot \nu+\omega^{2} \rho_{f} \nabla u h \cdot \nu- \\
& \nabla(\nabla p) h \cdot \nu-\nabla(\nabla g) h \cdot \nu- \\
& \left(\omega^{2} \rho_{f} u-\nabla p-\nabla g\right) \cdot\left[h^{\prime}\right]^{t} \nu=0 \quad \text { in } \Gamma,
\end{aligned}
$$

which concludes the proof of Lemma. 5.16.

Note that the proofs of Proposition 5.15 and Proposition 5.16 rely on the fact that $\frac{\partial \varphi_{\theta}}{\partial \theta}(0) h$ and $\frac{\partial \Psi_{\theta}}{\partial \theta}(0) h$ are well-defined. This can be easily verified in the case where $\Gamma$ is regular enough, i.e., $\mathcal{C}^{k}, k \geq 2$. The case where $\Gamma$ is assumed to be only a Lipschitz continuous polygonal-shaped boundary needs more careful considerations, as demonstrated in the following.

5.2. Extension to Lipschitz polygonal-shaped boundaries. The goal here is to prove that the transmission boundary conditions given by Eq. (5.50) and Eq. (5.73) are well-defined in the case where $\Gamma$ is assumed to be a Lipschitz continuous polygonalshaped boundary. To this end, we proceed by analyzing separately each term of the functions $F$ and $G$ given by Eqs. (3.7)-(3.8).

The following two properties are direct consequences of the classical trace theorems applied to the solution $(u, p)$ of the direct problem BVP (1) for $\theta=0$.

Property 5.17. Let $(u, p)$ be the solution of $B V P(1)$ for $\theta=0$ and $h \in \mathcal{C}^{2}\left(\mathbb{R}^{2}\right)$. Then, we have:
i. $p\left[h^{\prime}\right]^{t} \nu_{\mid \Gamma} \in\left(L^{2}(\Gamma)\right)^{2}$.
ii. $u \cdot\left[h^{\prime}\right]^{t} \nu_{\mid \Gamma} \in L^{2}(\Gamma)$,

where $\left[h^{\prime}\right]^{t}$ is the transpose Jacobian matrix of $h$.

Proof. First, we prove property i. First, recall that $p \in H_{\text {loc }}^{3 / 2}\left(\Omega^{f}\right)[26,38]$. Note that this result is an immediate consequence of Jerison-Kenig Lemma [26]. Then, we 
have $p_{\mid \Gamma} \in H^{1 / 2}(\Gamma)$. Moreover, because $h \in\left(\mathcal{C}^{2}\left(\mathbb{R}^{2}\right)\right)^{2}$, each entry of its Jacobian matrix $\left[h^{\prime}\right]$ is continuous. Since $\nu_{\mid \Gamma} \in\left(L^{\infty}(\Gamma)\right)^{2}$, the vector $\left[h^{\prime}\right]^{t} \nu_{\mid \Gamma}$ defines a vector of $\left(L^{\infty}(\Gamma)\right)^{2}$, which is a multiplier of $L^{2}(\Gamma)$. Hence, we must have $p\left[h^{\prime}\right]^{t} \nu_{\mid \Gamma} \in\left(L^{2}(\Gamma)\right)^{2}$.

Next, we prove property ii. Since $u \in\left(H^{3 / 2}\left(\Omega^{s}\right)\right)^{2}$, then $u_{\mid \Gamma} \in\left(H^{1 / 2}(\Gamma)\right)^{2}$. In addition, we have shown in the proof of property i that $\left[h^{\prime}\right]^{t} \nu_{\mid \Gamma} \in\left(L^{\infty}(\Gamma)\right)^{2}$. Then, we deduce that $u \cdot\left[h^{\prime}\right]^{t} \nu_{\mid \Gamma} \in L^{2}(\Gamma)$.

The next properties result from Proposition 4.5 and Proposition 4.13.

Property 5.18. Let $(u, p)$ be the solution of BVP (1) for $\theta=0$ and $\left(u^{\prime}, p^{\prime}\right)$ its corresponding Fréchet derivative at $\theta=0$ in the direction $h \in\left(\mathcal{C}^{2}\left(\mathbb{R}^{2}\right)\right)^{2}$. For $j=1, \ldots, M$, we have:
i. $p^{\prime} \nu_{\mid \Gamma_{j}} \in \tilde{\mathbf{H}}^{-1 / 2}\left(\Gamma_{j}\right)$.
ii. $\sigma\left(u^{\prime}\right) \nu_{\mid \Gamma_{j}} \in \tilde{\mathbf{H}}^{-3 / 2}\left(\Gamma_{j}\right)$.
iii. $\frac{\partial p^{\prime}}{\partial \nu} \mid \Gamma_{j} \in \tilde{H}^{-3 / 2}\left(\Gamma_{j}\right)$.
iv. $u^{\prime} \cdot \nu_{\mid \Gamma_{j}} \in \tilde{H}^{-1 / 2}\left(\Gamma_{j}\right)$.

Proof. First, we prove property i. Since $p \in H_{l o c}^{3 / 2}\left(\Omega^{f}\right)$, then $p^{\prime} \in H_{l o c}^{1 / 2}\left(\Omega^{f}\right)$ $[33,40,23]$. Moreover, since $p^{\prime}$ satisfies the Helmholtz equation (5.37), then we have $p^{\prime} \in D\left(\Delta, \Omega^{f}\right)$. Consequently, we can apply Proposition 4.5 and conclude that $p_{\mid \Gamma_{j}}^{\prime} \in \tilde{H}^{-1 / 2}\left(\Gamma_{j}\right)$. Additionally, we know that $\nu$ is locally regular. Hence, we necessarily have $p^{\prime} \nu_{\mid \Gamma_{j}} \in \tilde{\mathbf{H}}^{-1 / 2}\left(\Gamma_{j}\right)$.

Next, we prove property ii. Since $u \in\left(H^{3 / 2}\left(\Omega^{s}\right)\right)^{2}$, then $u^{\prime} \in\left(H^{1 / 2}\left(\Omega^{s}\right)\right)^{2}$. On the other hand, $u^{\prime}$ also satisfies Eq. (5.38). Therefore, $u^{\prime} \in D\left(\nabla \cdot \sigma, \Omega^{s}\right)$. Consequently, we can apply Proposition 4.13 and conclude that $\sigma\left(u^{\prime}\right) \nu_{\mid \Gamma_{j}} \in \tilde{\mathbf{H}}^{-3 / 2}\left(\Gamma_{j}\right)$.

property iii is a corollary of Proposition 4.5.

Finally, we prove property iv. From Proposition 4.13, we have: $u_{\mid \Gamma_{j}} \in \tilde{\mathbf{H}}^{-1 / 2}\left(\Gamma_{j}\right)$. Moreover, since $\nu$ is regular on $\Gamma_{j}$, then we must have that $u^{\prime} \cdot \nu_{\mid \Gamma_{j}} \in \tilde{H}^{-1 / 2}\left(\Gamma_{j}\right)$.

Next, we use again Proposition 4.5 and Proposition 4.13 to show that $(\nabla p \cdot h \nu)_{\mid \Gamma}$, $\left(\nabla p \cdot\left[h^{\prime}\right]^{t} \nu\right)_{\mid \Gamma},(\nabla u h \cdot \nu)_{\mid \Gamma}$, and $\sigma(u)\left[h^{\prime}\right]^{t} \nu_{\mid \Gamma}$ are well-defined. To this end, for simplicity purposes, we denote by $\partial$ one of the partial derivatives $\frac{\partial}{\partial x_{j}}, 1 \leq j \leq 2$. Moreover, we denote by $\left(\tilde{H}^{-1 / 2}\left(\Gamma_{j}\right)\right)^{2 \times 2}$ the space of square matrices of order 2 whose entries are in $\tilde{H}^{-1 / 2}\left(\Gamma_{j}\right)$.

Property 5.19. Let $(u, p)$ be the solution of $B V P$ (1) for $\theta=0$. Then, for $j=1, \ldots, M$, we have:
i. $\nabla p \cdot h \nu_{\mid \Gamma_{j}} \in \tilde{\mathbf{H}}^{-1 / 2}\left(\Gamma_{j}\right)$.
ii. $\nabla p \cdot\left[h^{\prime}\right]^{t} \nu_{\mid \Gamma_{j}} \in \tilde{H}^{-1 / 2}\left(\Gamma_{j}\right)$.
iii. $\nabla u h \cdot \nu_{\mid \Gamma_{j}} \in \tilde{H}^{-1 / 2}\left(\Gamma_{j}\right)$. 
iv. $\sigma(u)\left[h^{\prime}\right]^{t} \nu_{\mid \Gamma_{j}} \in \tilde{\mathbf{H}}^{-1 / 2}\left(\Gamma_{j}\right)$.

Proof. First, we prove property i. Since $p$ satisfies the Helmholtz equation given by Eq. (b) in BVP (1) and using the fact that the partial derivatives of $p$ commute with the Laplace operator, then we have:

$$
\Delta(\partial p)+k^{2} \partial p=0 .
$$

Observe that $\partial p$ is in $H_{l o c}^{1 / 2}\left(\Omega^{f}\right)$ because $p \in H_{l o c}^{3 / 2}\left(\Omega^{f}\right)$. Thus, using Eq. (5.87), we have $\partial p \in D\left(\Delta, \Omega^{f}\right)$. Hence, Proposition 4.5 ensures that $\partial p_{\mid \Gamma_{j}} \in \tilde{H}^{-1 / 2}\left(\Gamma_{j}\right)$, and therefore $\nabla p_{\mid \Gamma_{j}} \in \tilde{\mathbf{H}}^{-1 / 2}\left(\Gamma_{j}\right)$. Using the fact that the direction $h \in\left(\mathcal{C}^{2}\left(\mathbb{R}^{2}\right)\right)^{2}, \nabla p \cdot h_{\mid \Gamma_{j}}$ remains in $\tilde{H}^{-1 / 2}\left(\Gamma_{j}\right)$. Then, since $\nu$ is locally regular, $\nabla p \cdot h \nu_{\mid \Gamma_{j}}$ is also in $\tilde{\mathbf{H}}^{-1 / 2}\left(\Gamma_{j}\right)$.

Second, we prove property ii. We have shown in proof of property i that $\nabla p_{\mid \Gamma_{j}} \in$ $\tilde{\mathbf{H}}^{-1 / 2}\left(\Gamma_{j}\right)$. Moreover, observe that each entry of $\left[h^{\prime}\right]$ is in $\mathcal{C}^{1}\left(\mathbb{R}^{2}\right)$. Hence, using the fact that $\nu$ is locally regular, we deduce that $\nabla p \cdot\left[h^{\prime}\right]^{t} \nu_{j \mid \Gamma_{j}}$ is also in $\tilde{H}^{-1 / 2}\left(\Gamma_{j}\right)$.

Third, we prove property iii. It follows from the fact that (a) $u$ satisfies the Navier equation given by Eq. (a) in BVP (1), and (b) the partial derivatives of $u$ commute with the elastodynamics operator, that:

$$
\nabla \cdot \sigma(\partial u)+\omega^{2} \rho_{s} \partial u=0 .
$$

Since $\partial u \in\left(H^{1 / 2}\left(\Omega^{s}\right)\right)^{2}$, then it follows from Eq. (5.88) that $\partial u \in D\left(\nabla \cdot \sigma, \Omega^{s}\right)$. Hence, it follows from Proposition 4.13 that $\partial u_{\mid \Gamma_{j}} \in \tilde{\mathbf{H}}^{-1 / 2}\left(\Gamma_{j}\right)$, and therefore $\nabla u_{\mid \Gamma_{j}} \in$ $\left(\tilde{H}^{-1 / 2}\left(\Gamma_{j}\right)\right)^{2 \times 2}$. Using the fact that $h \in\left(\mathcal{C}^{2}\left(\mathbb{R}^{2}\right)\right)^{2}$ and $\nu$ is locally regular, we deduce that $\nabla u h \cdot \nu_{\mid \Gamma_{j}}$ is also in $\tilde{H}^{-1 / 2}\left(\Gamma_{j}\right)$.

Last, we prove property iv. We have shown in proof of property iii that $\partial u_{\mid \Gamma_{j}} \in$ $\tilde{\mathbf{H}}^{-1 / 2}\left(\Gamma_{j}\right)$. Consequently, the trace of the strain tensor $\varepsilon(u) \in\left(\tilde{H}^{-1 / 2}\left(\Gamma_{j}\right)\right)^{2 \times 2}$. Using Eq. (2.2), we conclude that the trace of the stress tensor $\sigma(u)_{\mid \Gamma_{j}}$ remains in $\left(\tilde{H}^{-1 / 2}\left(\Gamma_{j}\right)\right)^{2 \times 2}$. Furthermore, since each entry of $\left[h^{\prime}\right]$ is in $\mathcal{C}^{1}\left(\mathbb{R}^{2}\right)$, and $\nu$ is locally regular, we deduce that $\sigma(u)\left[h^{\prime}\right]^{t} \nu_{\mid \Gamma_{j}} \in \tilde{\mathbf{H}}^{-1 / 2}\left(\Gamma_{j}\right)$.

The proof of Theorem 3.2 is completed after proving that the high-order derivative terms $(\nabla(\nabla p) h) \cdot \nu_{\mid \Gamma}$ and $h^{t} \nabla \sigma(u) \nu_{\mid \Gamma}$ are also well-defined.

Property 5.20. Let $(u, p)$ be the solution of $B V P$ (1) for $\theta=0$. For $j=$ $1, \ldots, M$, we have:

i. $h^{t} \nabla(\nabla p) \nu_{\mid \Gamma_{j}} \in \tilde{H}^{-3 / 2}\left(\Gamma_{j}\right)$.

ii. $\left(h^{t} \nabla \sigma_{l}(u) \nu_{\mid \Gamma_{j}}\right)_{1 \leq l \leq 2} \in \tilde{H}^{-3 / 2}\left(\Gamma_{j}\right)$.

Proof. We first prove property i. We have already shown that $\partial p \in D\left(\Delta, \Omega^{f}\right)$ (see the proof of Property 5.19). Therefore, Proposition 4.5 ensures that $\nabla(\partial p) \cdot \nu_{\mid \Gamma_{j}}$ is in $\tilde{H}^{-3 / 2}\left(\Gamma_{j}\right)$. Consequently, $\nabla(\nabla p) \nu_{\mid \Gamma_{j}}$ is in $\tilde{\mathbf{H}}^{-3 / 2}\left(\Gamma_{j}\right)$. Moreover, since $h_{\mid \Gamma_{j}} \in$ $\left(\mathcal{C}^{2}\left(\Gamma_{j}\right)\right)^{2}$, we deduce that:

$$
h^{t} \nabla(\nabla p) \nu_{\mid \Gamma_{j}} \in \tilde{H}^{-3 / 2}\left(\Gamma_{j}\right) .
$$

Next, we prove property ii. We have shown that $\partial u \in D\left(\nabla \cdot \sigma, \Omega^{s}\right)$ (see the proof of Property 5.19). Therefore, Proposition 4.13 ensures that $\sigma(\partial u) \nu_{\mid \Gamma_{j}} \in \tilde{\mathbf{H}}^{-3 / 2}\left(\Gamma_{j}\right)$. 
Consequently, $\partial \sigma_{l}(u) \nu_{\mid \Gamma_{j}} \in \tilde{H}^{-3 / 2}\left(\Gamma_{j}\right)$, for all $1 \leq l \leq n$. Hence, $\nabla \sigma_{l}(u) \nu_{\mid \Gamma_{j}} \in$ $\tilde{\mathbf{H}}^{-3 / 2}\left(\Gamma_{j}\right)$. Moreover, since $h_{\mid \Gamma_{j}} \in\left(\mathcal{C}^{2}\left(\Gamma_{j}\right)\right)^{2}$, we conclude that:

$$
\left(h^{t} \nabla \sigma_{l}(u) \nu_{\mid \Gamma_{j}}\right)_{1 \leq l \leq 2} \in \tilde{H}^{-3 / 2}\left(\Gamma_{j}\right) .
$$

Remark 5.21. We must emphasize on the important role of the local regularity of the unit normal vector in the previous proofs. Indeed, this regularity property was crucial to establish the well-definedness on $\Gamma$ of quantities such as $p^{\prime} \nu$ and $u^{\prime} \cdot \nu$.

6. Summary \& Conclusion. We have proved that the Fréchet derivative of the elasto-acoustic scattered field with respect to Lipschitz continuous polygonalshaped domains is the solution of the same elasto-acoustic scattering problem but with different right-hand sides in the boundary conditions. From a computational viewpoint, this result is relevant to the solution of inverse obstacle problems with regularized iterative methods. Indeed, the scatterers are transformed into polygonalshaped domains when employing finite element methods for solving the corresponding direct scattering problems. In addition, all entries of the Jacobians are evaluated, at each iteration, by solving the same linear algebraic system with multiple right-hand sides. From a mathematical viewpoint, the proposed proof can be extended to threedimensional problems, i.e., scatterers whose domains are polyhedra.

Acknowledgements. The authors acknowledge the support of the European Union's Horizon H2020, research and innovation program under the Marie SklodowskaCurie grant agreement \# 644202. They also thank the anonymous Referees for their remarks and suggestions. Any opinions, findings, conclusions, or recommendations expressed in this material are those of the authors and do not necessarily reflect the views of funding agency.

\section{REFERENCES}

[1] R. A. Adams, Sobolev spaces, Academic Press, New-York, 1975.

[2] F. Assous and P. Ciarlet JR, Une caractérisation de l'orthogonal de $\Delta\left(H^{2}(\Omega) \cap H_{0}^{1}(\Omega)\right)$ dans $L^{2}(\Omega)$, C. R. Acad. Sci. Paris Sér. I Math., 325 (1997), pp. 605-610.

[3] F. Assous, P. Ciarlet JR, and E. Garcia, Singular electromagnetic fields: inductive approach, C. R. Acad. Sci. Paris Sér. I Math., 341 (2005), pp. 605-610.

[4] F. Assous, P. Ciarlet JR, And E. Garcia, A characterization of singular electromagnetic fields by an inductive approach, Int. J. Numer. Anal. Mod., 5 (2008), pp. 491-515.

[5] H. BarucQ, R. Djellouli, and E. Estechanandy, Characterization of the Fréchet Derivative of the Elasto-Acoustic Field with respect to Lipschitz Domains, Journal of Inverse and Ill-Posed Problems, 22 (2014), pp. 1-8. DOI:10.1515/jip-2012-0098.

[6] H. Barucq, R. Djellouli, and E. Estecahandy, On the existence and the uniqueness of the solution of a fluid-structure interaction scattering problem, Journal of Mathematical Analysis and Applications, 412 (2014), pp. 571-588.

[7] H. Barucq, R. Djellouli, and E. Estecahandy, Fréchet differentiability of the elastoacoustic scattered field with respect to Lipschitz domains, Mathematical Methods in the Applied Sciences, (2015), doi:10.1002/mma.3444.

[8] D. Colton And R. KRess, Inverse acoustic and electromagnetic scattering theory, Berlin, 1992.

[9] M. Costabel And F. Le Louër, Shape Derivatives of Boundary Integral Operators in Electromagnetic Scattering. Part I: Shape Differentiability of Pseudo-homogeneous Boundary Integral Operators, Integral Equations and Operator Theory, 72 (2012), pp. 509-535. 
[10] M. Costabel And F. Le Louër, Shape Derivatives of Boundary Integral Operators in Electromagnetic Scattering. Part II: Application to Scattering by a Homogeneous Dielectric Obstacle, Integral Equations and Operator Theory, 73 (2012), pp. 17-48.

[11] R. Djellouli, Inverse acoustic problems, in Computational Methods for Acoustic Problems, Saxe-Coburg Publications, 2008.

[12] R. Djellouli and C. Farhat, On the Characterization of the Fréchet Derivative with Respect to a Lipschitz Domain of the Acoustic Scattered Field, J. Math. Anal. Appl., 238 (1999), pp. 259-276.

[13] R. Djellouli, C. Farhat, J. Mandel, and P. VanĚK, Continuous Fréchet Differentiability With Respect To Lipschitz Domain And A Stability Estimate For Direct Acoustic Scattering Problems, IMA J. Appl. Math., 63 (1999), pp. 51-69.

[14] E. Estecahandy, Contribution l'analyse mathématique et la résolution numérique d'un problème inverse de scattering élasto-acoustique, $\mathrm{PhD}$ thesis, Université de Pau et des Pays de l'Adour, 2013. Available online at: https://tel.archives-ouvertes.fr/tel-00880628.

[15] L. C. Evans, Partial Differential Equations, American Mathematical Society, Providence R.I., 1993.

[16] G. Geymonat and F. Krasucki, On the existence of the Airy function in Lipschitz domains. Application to the traces of $H^{2}$, C. R. Acad. Sci. Paris Sér. I Math., 330 (2000), pp. 355360.

[17] S. F. Gilyazov and N. L. Golóman, Regularization of ill-posed problems by iteration methods, Dordrecht: Kluwer Academic Publishers, 2000.

[18] P. Grisvard, Alternative de Fredholm relative au problème de Dirichlet dans un polygone ou un polyèdre, Bollettino dell'Unione Mathematica Italiana, 4 (1972), pp. 132-164.

[19] P. Grisvard, Elliptic Problems in Nonsmooth Domains, London, 1985.

[20] P. Grisvard, Singularities in Boundary Problems, Paris, 1992.

[21] H. Haddar And R. Kress, On the Fréchet derivative for Obstacle Scattering with an Impedance Boundary Condition, Siam J. Appl. Math., 65 (2004), pp. 194-208.

[22] T. HARGÉ, Valeurs propres d'un corps élastique, C. R. Acad. Sci. Paris Sér. I Math., 311 (1990), pp. 857-859.

[23] A. Henrot and M. Pierre, Variation et optimisation de formes: une analyse géométrique, Springer, SMAI, 2005.

[24] F. Hettlich, Fréchet derivatives in inverse obstacle scattering, Inverse Problems, 11 (1995), pp. 371-382.

[25] T. Hohage, Iterative Methods in Inverse Obstacle Scattering: Regularization Theory of Linear and Nonlinear Exponentially Ill-Posed Problems, PhD thesis, PhD in Numerical analysis, University of Linz, 1999.

[26] D. S. Jerison And C. E. Kenig, The Neumann problem on Lipschitz domains, Bull. Amer. Math. Sot, 4 (1981), pp. 203-207.

[27] D. S. Jones, Low-frequency scattering by a body in lubricated contact, Quart. J. Mech. Appl. Math., 36 (1983), pp. 111-138.

[28] M. C. Junger And D. Feit, Sound, Structures, and Their Interaction, The MIT Press, Cambridge, MA, 1972.

[29] A. KIRSCH, The domain derivative and two applications in inverse scattering theory, Inverse Problems, 9 (1993), pp. 81-96.

[30] F. LE LoUËR, Optimisation de formes dantennes lentilles intégrées aux ondes millimétriques, $\mathrm{PhD}$ thesis, PhD in Numerical analysis, University of Rennes 1, 2009.

[31] J. L. Lions, Problèmes aux limites dans les équations aux dérivées partielles, Montreal, 1965.

[32] C. J. Luke And P. A. Martin, Fluid-Solid Interaction: Acoustic Scattering by a Smooth Elastic Obstacle, SIAM J. Appl. Math., 55 (1995), pp. 904-922.

[33] F. Murat and S. J., Sur le contrôle par un domaine géométrique. Rapport du L.A., 1976.

[34] J. NeČAs, Les méthodes directes en théorie des équations elliptiques, Paris, 1967.

[35] R. Pотthast, Fréchet differentiability of boundary integral operators in inverse acoustic scattering, Inverse Problems, 10 (1994), pp. 431-447.

[36] R. Potthast, Domain derivatives in electromagnetic scattering, Math. Methods Appl. Sci., 19 (1996), pp. 1157-1175.

[37] R. Potthast, Fréchet-differentiability of the solution to the acoustic Neumann scattering problem with respect to the domain, J. Inverse Ill-Posed Probl., 4 (1996), pp. 67-84.

[38] J. Sanchez-Hubert and E. Sanchez-Palencia, Vibration and Coupling of Continuous Systems, Springer Verlag, New York, 1989.

[39] S. Sauter and C. Schwab, Boundary Element Methods, Springer Series in Computational Mathematics, 2011.

[40] J. Simon, Differentiation with respect to the domain in boundary value problems, Numer. Funct. 
Anal. Optim., 2 (1980), pp. 649-687.

[41] K. YosidA, Functional Analysis, Springer, Berlin, 1964. 